\title{
Oxidative Radical Addition-Cyclization of Sulfonyl Hydrazones with Simple Olefins by Binary Acid Catalysis
}

Xingren Zhong, ${ }^{\dagger}$ Jian Lv, ${ }^{*}+,+$, and Sanzhong Luo ${ }^{*,+, *}$

${ }^{\dagger}$ Beijing National Laboratory for Molecular Sciences (BNLMS), Key Laboratory of Molecular Recognition and Function, Institute of Chemistry, Chinese Academy of Sciences, Beijing 100190, China; University of Chinese Academy of Sciences, Beijing, 100049, China.

* Collaborative Innovation Center of Chemical Science and Engineering (Tianjin), Tianjin 300071, China.

TABLE OF CONTENTS

PAGE

General Experimental S2

Oxidative Radical [4+2] cycloaddition S3

Mechanistic Studies of Binary-acid Catalysis S15

NMR spectrum S27 


\section{General Experimental}

Commercial reagents were used as received, unless otherwise indicated. Nuclear magnetic resonance (NMR) spectra were recorded using Bruker AV-400 and AV-500 spectrometers. ${ }^{1} \mathrm{H}$ and ${ }^{13} \mathrm{C}$, NMR spectra were measured on a NMR instrument (400 $\mathrm{MHz}$ and $500 \mathrm{MHz}$ for ${ }^{1} \mathrm{H}$ NMR, 100 or $125 \mathrm{MHz}$ for ${ }^{13} \mathrm{C}$ NMR). Tetramethylsilane (TMS) served as the internal standard for ${ }^{1} \mathrm{H} \mathrm{NMR}$, and $\mathrm{CDCl}_{3}$ served as the internal standard for ${ }^{13} \mathrm{C}$ NMR. The following abbreviations were used to express the multiplicities: $\mathrm{s}=$ singlet; $\mathrm{d}=$ doublet; $\mathrm{t}=$ triplet; $\mathrm{q}=$ quartet; $\mathrm{m}=$ multiplet; $\mathrm{br}=$ broad . HRMS was recorded on a commercial apparatus (ESI).

Sulfonyl hydrazones 2 were prepared according to literature procedure. ${ }^{[1]}$ Simple olefins (3a-j, 3l) and ketones (6a-f) were received from commercial sources without further purification, except for 2-vinylthiophene 3k, which was prepare according to the literature procedure. ${ }^{[2]}$ Sulfonyl hydrazines 5a, b were received from commercial sources without further purification, $\mathbf{5 c}$, $\mathbf{d}$ were prepared according to the literature procedure. ${ }^{[3]}$ Solvents were freshly dried according to the Purification Handbook Purification of Laboratory Chemicals before using. 


\section{Oxidative Radical [4+2] Cycloaddition}

Table S1. Influence of reaction parameters on the catalytic oxidative [4+2] cycloaddition of N-tosylhydrazones 2a and styrene 3a.

\begin{tabular}{|c|c|c|c|c|}
\hline entry & Lewis acid/1 $\mathbf{1}$ & solvent & $t(\mathrm{~h})$ & yield $(\%)^{b}$ \\
\hline 1 & $\mathrm{Cu}\left(\mathrm{CH}_{3} \mathrm{CN}\right)_{4} \mathrm{BF}_{4}$ & $\mathrm{CH} 3 \mathrm{CN}$ & 6 & 63 \\
\hline 2 & $\mathrm{CuX}(\mathrm{X}=\mathrm{Cl}, \mathrm{Br}$, or I $)$ & $\mathrm{CH}_{3} \mathrm{CN}$ & 6 & $<20$ \\
\hline 3 & $\mathrm{Cu}(\mathrm{OTf})_{2}$ & $\mathrm{CH}_{3} \mathrm{CN}$ & 6 & 9 \\
\hline 4 & $\operatorname{In}(\mathrm{OTf})_{3}$ & $\mathrm{CH}_{3} \mathrm{CN}$ & 6 & 1 \\
\hline 5 & $\mathrm{Mg}(\mathrm{OTf})_{2}$ & $\mathrm{CH}_{3} \mathrm{CN}$ & 6 & 3 \\
\hline 6 & $\mathrm{Zn}(\mathrm{OTf})_{2}$ & $\mathrm{CH}_{3} \mathrm{CN}$ & 6 & 3 \\
\hline 7 & $\mathrm{FeCl}_{3}$ & $\mathrm{CH}_{3} \mathrm{CN}$ & 6 & 6 \\
\hline 8 & $\mathrm{FeBr}_{2}$ & $\mathrm{CH}_{3} \mathrm{CN}$ & 6 & NR \\
\hline $9^{d}$ & $\mathrm{Cu}\left(\mathrm{CH}_{3} \mathrm{CN}\right)_{4} \mathrm{BF}_{4}$ & $\mathrm{CH}_{3} \mathrm{CN}$ & 6 & 32 \\
\hline
\end{tabular}

${ }^{a}$ General conditions: $\mathrm{Cu}\left(\mathrm{CH}_{3} \mathrm{CN}\right)_{4} \mathrm{BF}_{4}(5 \mathrm{~mol} \%), 1$ (5 mo 1\%), $\mathrm{I}_{2}(5 \mathrm{~mol} \%)$, TBHP $(0.8 \mathrm{mmol}), 2 \mathrm{a}(0.2 \mathrm{mmol})$, and 3a $(1.0 \mathrm{mmol})$ at room temperature. ${ }^{b}$ Determined by ${ }^{1} \mathrm{H}$ NMR analysis with an internal standard, 1,3,5-trimethyloxylbenzene. ${ }^{c} \mathbf{1}(10 \mathrm{~mol} \%) .{ }^{d}$ TBHP $(0.4 \mathrm{mmol})$. Tf $=$ Trifluoro-methanesulfonate. NR $=$ No reaction 


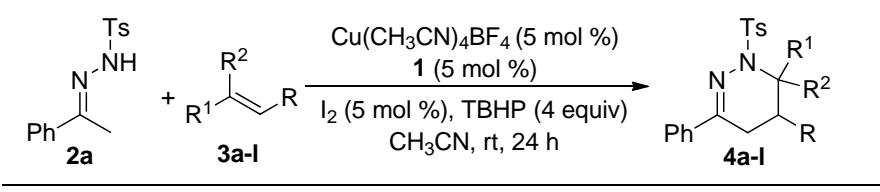

General procedure for oxidative [4+2] cycloaddition of sulfonyl hydrazones 2a

with simple olefins 3: To an oven-dried reaction tube was added $\mathrm{Cu}\left(\mathrm{CH}_{3} \mathrm{CN}\right)_{4} \mathrm{BF}_{4}$ (0.01 mmol, $5 \mathrm{~mol} \%)$ and diphenyl phosphate 1 (0.01 mmol, $5 \mathrm{~mol} \%)$. Distilled anhydrous $\mathrm{CH}_{3} \mathrm{CN}(0.5 \mathrm{~mL})$ was added. After stirring for $0.5 \mathrm{~h}$, sulfonyl hydrazine 2a (0.2 mmol, in $0.4 \mathrm{~mL} \mathrm{CH} 3 \mathrm{CN})$, simple olefin 3 (1.0 mmol), $\mathrm{I}_{2}(5 \mathrm{~mol} \%$, in $0.1 \mathrm{~mL}$ $\left.\mathrm{CH}_{3} \mathrm{CN}\right)$, and TBHP (0.8 mmol in decane) were added successively to the stirring reaction mixture. The mixture was stirred for 12-24 hours at room temperature. Purification of mixture by column chromatography on silica gel (petroleum ether : ethyl acetate $=20: 1$ to $10: 1$ as eluent) gave $\mathbf{4 a - 1}, \mathbf{4} \mathbf{u}$.<smiles>[CH2]C1=NN([12F])C(c2ccccc2)CC1</smiles>

$67.9 \mathrm{mg}$, yield: $87 \% ;{ }^{1} \mathrm{H}$ NMR (400 MHz, $\left.\mathrm{CDCl}_{3}\right): \delta 7.74-7.72(\mathrm{~m}, 2 \mathrm{H}), 7.68(\mathrm{~d}, J=$ 8.3 Hz, 2H), 7.39-7.33 (m, 3H), 7.19-7.13 (m, 5H), 6.98-6.96 (m, 2H), $5.64(\mathrm{~s}, 1 \mathrm{H})$, 2.61-2.52 (m, 1H), $2.36(\mathrm{~s}, 3 \mathrm{H}), 2.17-2.04(\mathrm{~m}, 3 \mathrm{H}) \mathrm{ppm} ;{ }^{13} \mathrm{C} \mathrm{NMR}\left(100 \mathrm{MHz}, \mathrm{CDCl}_{3}\right)$ : $\delta 147.3,143.5,140.3,137.0,136.0,129.2,128.5,128.4,128.1,127.3,126.1,125.3$, 55.2, 24.9, 21.6, 17.9 ppm; IR (KBr, $\left.\mathrm{cm}^{-1}\right)$ : 3062, 3029, 2929, 1598, 1495, 1448, 1356, 894, 754, 695, 672; HRMS (ESI) calcd for $\mathrm{C}_{23} \mathrm{H}_{23} \mathrm{~N}_{2} \mathrm{O}_{2} \mathrm{~S}^{+}(\mathrm{M}+\mathrm{H})^{+} 391.1475$, found 391.1469.

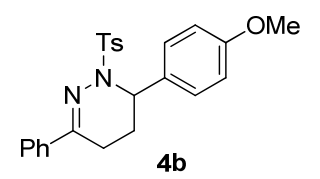

$49.7 \mathrm{mg}$, yield: 59\%; ${ }^{1} \mathrm{H}$ NMR (400 MHz, $\left.\mathrm{CDCl}_{3}\right): \delta 7.74-7.72(\mathrm{~m}, 2 \mathrm{H}), 7.67(\mathrm{~d}, J=$ $8.2 \mathrm{~Hz}, 2 \mathrm{H}), 7.39-7.34(\mathrm{~m}, 3 \mathrm{H}), 7.17$ (d, $J=8.2 \mathrm{~Hz}, 2 \mathrm{H}), 6.89$ (d, J=8.7 Hz, 2H), $6.69(\mathrm{~d}, J=8.6 \mathrm{~Hz}, 2 \mathrm{H}), 5.59$ (s, 1H), $3.75(\mathrm{~s}, 3 \mathrm{H}), 2.61-2.56(\mathrm{~m}, 1 \mathrm{H}), 2.37$ (s, 3H), 2.19-2.04 (m, 3H) ppm; ${ }^{13} \mathrm{C}$ NMR (100 MHz, $\left.\mathrm{CDCl}_{3}\right): \delta 158.8,147.2,143.4,137.0$, $136.0,132.5,129.1,128.4,128.1,127.3,125.3,113.8,55.3,54.7,25.1,21.6,17.9$ 
ppm; IR (KBr, cm $\left.{ }^{-1}\right): 3067,2956,2929,2836,1612,1356,1251,1095,897,758,694$, 578, 548; HRMS (ESI) calcd for $\mathrm{C}_{24} \mathrm{H}_{25} \mathrm{~N}_{2} \mathrm{O}_{3} \mathrm{~S}^{+}(\mathrm{M}+\mathrm{H})^{+} 421.1580$, found 421.1575 .

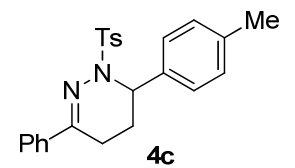

$61.9 \mathrm{mg}$, yield: 77\%; ${ }^{1} \mathrm{H}$ NMR (400 MHz, $\left.\mathrm{CDCl}_{3}\right): \delta$ 7.73-7.68 (m, 4H), 7.38-7.33 (m, 3H), $7.17(\mathrm{~d}, J=8.1 \mathrm{~Hz}, 2 \mathrm{H}), 6.97(\mathrm{~d}, J=7.9 \mathrm{~Hz}, 2 \mathrm{H}), 6.87$ (d, $J=7.9 \mathrm{~Hz}, 2 \mathrm{H}), 5.60$ (s, 1H), 2.60-2.54 (m, 1H), $2.37(\mathrm{~s}, 3 \mathrm{H}), 2.28(\mathrm{~s}, 3 \mathrm{H}), 2.17-2.02(\mathrm{~m}, 3 \mathrm{H}) \mathrm{ppm} ;{ }^{13} \mathrm{C}$ NMR (100 MHz, $\left.\mathrm{CDCl}_{3}\right): \delta 147.2,143.5,137.4,137.1,137.0,136.0,129.2,129.1$, 128.4, 128.1, 126.0, 125.3, 55.0, 24.9, 21.6, 21.0, 17.9 ppm; IR $\left(\mathrm{KBr}, \mathrm{cm}^{-1}\right): 3056$, 3029, 2924, 2862, 1597, 1514, 1447, 987, 938, 898, 757, 694; HRMS (ESI) calcd for $\mathrm{C}_{24} \mathrm{H}_{25} \mathrm{~N}_{2} \mathrm{O}_{2} \mathrm{~S}^{+}(\mathrm{M}+\mathrm{H})^{+}$405.1631, found 405.1624.

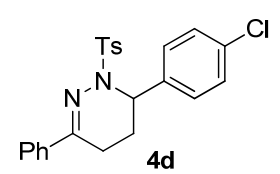

70.9 mg, yield: 84\%; ${ }^{1} \mathrm{H}$ NMR (400 MHz, $\left.\mathrm{CDCl}_{3}\right): \delta$ 7.73-7.69 (m, 4H), 7.39-7.35 (m, 3H), $7.20(\mathrm{~d}, J=8.1 \mathrm{~Hz}, 2 \mathrm{H}), 7.14(\mathrm{~d}, J=8.5 \mathrm{~Hz}, 2 \mathrm{H}), 6.93(\mathrm{~d}, J=8.4 \mathrm{~Hz}, 2 \mathrm{H}), 5.61$ $(\mathrm{s}, 1 \mathrm{H}), 2.63-2.54(\mathrm{~m}, 1 \mathrm{H}), 2.38(\mathrm{~s}, 3 \mathrm{H}), 2.14-2.03(\mathrm{~m}, 3 \mathrm{H}) \mathrm{ppm} ;{ }^{13} \mathrm{C} \mathrm{NMR}(100 \mathrm{MHz}$, $\left.\mathrm{CDCl}_{3}\right): \delta 147.4,143.8,138.9,136.8,135.8,133.2,129.3,128.6,128.5,128.0,127.5$, 125.3, 54.6, 24.8, 21.6, 17.8 ppm; IR (KBr, cm $\left.{ }^{-1}\right)$ : 3065, 2929, 2859, 1597, 1492 , 1358, 1171, 1094, 1014, 939, 898, 756, 681, 575; HRMS (ESI) calcd for $\mathrm{C}_{23} \mathrm{H}_{22} \mathrm{ClN}_{2} \mathrm{O}_{2} \mathrm{~S}^{+}(\mathrm{M}+\mathrm{H})^{+} 425.1085$, found 425.1077 .

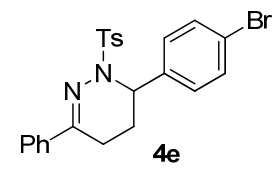

$74.5 \mathrm{mg}$, yield: 80\%; ${ }^{1} \mathrm{H} \mathrm{NMR}\left(400 \mathrm{MHz}, \mathrm{CDCl}_{3}\right)$ : $\delta$ 7.73-7.69 (m, 4H), 7.39-7.35 (m, 3H), 7.29 (d, $J=8.5 \mathrm{~Hz}, 2 \mathrm{H}), 7.20(\mathrm{~d}, J=8.1 \mathrm{~Hz}, 2 \mathrm{H}), 6.87$ (d, $J=8.4 \mathrm{~Hz}, 2 \mathrm{H}), 5.59$ (s, 1H), 2.63-2.54 (m, 1H), 2.39 (s, 3H), 2.14-2.03 (m, 3H) ppm; ${ }^{13} \mathrm{C}$ NMR (100 MHz, $\left.\mathrm{CDCl}_{3}\right): \delta 147.4,143.8,139.5,136.8,135.8,131.6,129.3,128.5,128.0,127.9,125.3$, 121.3, 54.7, 24.7, 21.6, 17.8 ppm; IR $\left(\mathrm{KBr}, \mathrm{cm}^{-1}\right): 3065,2929,2862,1596,1488$, 
1010, 939, 898, 771, 693, 644; HRMS (ESI) calcd forC $\mathrm{C}_{23} \mathrm{H}_{22} \mathrm{BrN}_{2} \mathrm{O}_{2} \mathrm{~S}^{+}(\mathrm{M}+\mathrm{H})^{+}$ 469.0580, found 469.0572 .

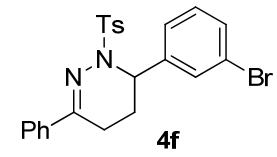

$78.5 \mathrm{mg}$, yield: 84\%; ${ }^{1} \mathrm{H}$ NMR (400 MHz, $\left.\mathrm{CDCl}_{3}\right)$ : $\delta$ 7.74-7.68 (m, 4H), 7.39-7.37 (m, 3H), $7.32(\mathrm{~d}, J=8.0 \mathrm{~Hz}, 1 \mathrm{H}), 7.20(\mathrm{~d}, J=8.2 \mathrm{~Hz}, 2 \mathrm{H}), 7.07-7.02(\mathrm{~m}, 2 \mathrm{H}), 6.93(\mathrm{~d}, J=$ $7.8 \mathrm{~Hz}, 1 \mathrm{H}), 5.60(\mathrm{~d}, J=3.2 \mathrm{~Hz}, 1 \mathrm{H}), 2.65-2.55(\mathrm{~m}, 1 \mathrm{H}), 2.39(\mathrm{~s}, 3 \mathrm{H}), 2.17-2.06(\mathrm{~m}$,

$3 \mathrm{H}) \mathrm{ppm} ;{ }^{13} \mathrm{C} \mathrm{NMR}\left(100 \mathrm{MHz}, \mathrm{CDCl}_{3}\right): \delta 147.4,143.9,142.5,136.9,135.7,130.6$, $130.1,129.3,129.3,129.1,128.4,128.0,125.4,124.9,122.6,54.6,24.8,21.6,17.8$ ppm; IR (KBr, cm $\left.{ }^{-1}\right)$ : 3065, 2924, 2854, 1595, 1093, 940, 899, 776, 756, 677, 583; HRMS (ESI) calcd for $\mathrm{C}_{23} \mathrm{H}_{22} \mathrm{BrN}_{2} \mathrm{O}_{2} \mathrm{~S}^{+}(\mathrm{M}+\mathrm{H})^{+} 469.0580$, found 469.0578 .

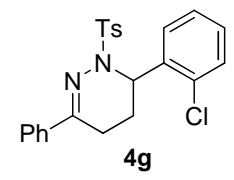

$42.5 \mathrm{mg}$, yield: 50\%; ${ }^{1} \mathrm{H}$ NMR (400 MHz, $\left.\mathrm{CDCl}_{3}\right): \delta$ 7.77-7.73 (m, 4H), 7.40-7.35 (m, 4H), $7.22(\mathrm{~d}, J=8.1 \mathrm{~Hz}, 2 \mathrm{H}), 7.18-7.13(\mathrm{~m}, 1 \mathrm{H}), 6.96-6.92(\mathrm{~m}, 1 \mathrm{H}), 6.76-6.74(\mathrm{~m}$, 1H), 5.97-5.96 (m, 1H), 2.64-2.59 (m, 1H), 2.39 (s, 3H), 2.35-2.30 (m, 1H), 2.09-1.92 $(\mathrm{m}, 2 \mathrm{H}) \mathrm{ppm} ;{ }^{13} \mathrm{C} \mathrm{NMR}\left(100 \mathrm{MHz}, \mathrm{CDCl}_{3}\right): \delta 146.8,143.7,137.6,136.8,135.9$, $131.1,130.0,129.3,129.3,128.7,128.4,128.2,128.1,126.7,125.3,52.9,22.2,21.6$, 17.9 ppm; IR (KBr, cm $\left.{ }^{-1}\right): 3065,2926,2865,1445,1359,1095,941,898,754,694$, 579; HRMS (ESI) calcd for $\mathrm{C}_{23} \mathrm{H}_{22} \mathrm{ClN}_{2} \mathrm{O}_{2} \mathrm{~S}^{+}(\mathrm{M}+\mathrm{H})^{+} 425.1085$, found 425.1079 .

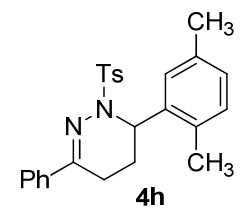

$76.3 \mathrm{mg}$, yield: 91\%; ${ }^{1} \mathrm{H}$ NMR (400 MHz, $\left.\mathrm{CDCl}_{3}\right): \delta$ 7.78-7.75 (m, 2H), $7.60(\mathrm{~d}, J=$ $8.3 \mathrm{~Hz}, 2 \mathrm{H}), 7.41-7.36(\mathrm{~m}, 3 \mathrm{H}), 7.13$ (d, $J=8.1 \mathrm{~Hz}, 2 \mathrm{H}), 7.03$ (d, $J=7.7 \mathrm{~Hz}, 1 \mathrm{H})$, $6.87(\mathrm{~d}, J=7.5 \mathrm{~Hz}, 1 \mathrm{H}), 6.13(\mathrm{~s}, 1 \mathrm{H}), 5.78(\mathrm{~s}, 1 \mathrm{H}), 2.65-2.60(\mathrm{~m}, 1 \mathrm{H}), 2.39(\mathrm{~s}, 3 \mathrm{H})$, $2.35(\mathrm{~s}, 3 \mathrm{H}), 2.22-2.03(\mathrm{~m}, 3 \mathrm{H}), 1.85$ (s, 3H) ppm; ${ }^{13} \mathrm{C} \mathrm{NMR}\left(100 \mathrm{MHz}, \mathrm{CDCl}_{3}\right): \delta$ $146.5,143.3,137.9,137.2,136.0,135.1,130.7,129.1$, 129.0, 128.4, 128.1, 128.0, 127.2, 125.4, 52.2, 22.9, 21.5, 20.9, 18.6, 17.7 ppm; IR (KBr, $\left.\mathrm{cm}^{-1}\right): 3026,2924,2868$, 
1597, 1446, 944, 808, 762, 693, 645, 582; HRMS (ESI) calcd for $\mathrm{C}_{25} \mathrm{H}_{27} \mathrm{~N}_{2} \mathrm{O}_{2} \mathrm{~S}^{+}$ $(\mathrm{M}+\mathrm{H})^{+}$419.1788, found 419.1782.

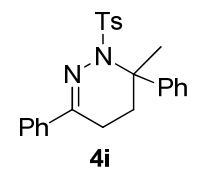

$75.0 \mathrm{mg}$, yield: 93\%; ${ }^{1} \mathrm{H}$ NMR (400 $\left.\mathrm{MHz}, \mathrm{CDCl}_{3}\right): \delta 7.85(\mathrm{~d}, J=8.1 \mathrm{~Hz}, 2 \mathrm{H})$, 7.69-7.67 (m, 2H), 7.38-7.34 (m, 6H), 7.28-7.26 (m, 4H), 2.57-2.49 (m,1H), $2.40(\mathrm{~s}$, $3 \mathrm{H}), 2.38-2.29(\mathrm{~m}, 1 \mathrm{H}), 2.16-2.09(\mathrm{~m}, 1 \mathrm{H}), 2.03(\mathrm{~s}, 3 \mathrm{H}), 2.01-1.95(\mathrm{~m}, 1 \mathrm{H}) \mathrm{ppm} ;{ }^{13} \mathrm{C}$ NMR (100 MHz, $\left.\mathrm{CDCl}_{3}\right): \delta 145.6,145.6,143.4,137.4,137.0,129.0,129.0,128.6$, $128.4,128.4,127.0,125.3,125.3,62.4,37.6,25.0,21.6,20.2 \mathrm{ppm}$; IR $\left(\mathrm{KBr}, \mathrm{cm}^{-1}\right)$ : 3062, 2924, 2854, 1597, 1494, 1446, 1087, 1037, 863, 781, 695, 565; HRMS (ESI) calcd for $\mathrm{C}_{24} \mathrm{H}_{25} \mathrm{~N}_{2} \mathrm{O}_{2} \mathrm{~S}^{+}(\mathrm{M}+\mathrm{H})^{+}$405.1631, found 405.1626.

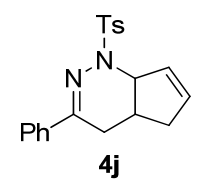

$69.9 \mathrm{mg}$, yield: 99\%; ${ }^{1} \mathrm{H}$ NMR (400 $\left.\mathrm{MHz}, \mathrm{CDCl}_{3}\right): \delta 7.95(\mathrm{~d}, J=8.2 \mathrm{~Hz}, 2 \mathrm{H})$, 7.69-7.66 (m, 2H), 7.37-7.32 (m, 5H), 5.92-5.87 (m, 2H), $4.76(\mathrm{~d}, J=7.7 \mathrm{~Hz}, 1 \mathrm{H})$, 2.69-2.63 (m, 2H), 2.44-2.37 (m, 4H), 2.19-2.10 (m, 2H) ppm; ${ }^{13} \mathrm{C}$ NMR (100 MHz, $\left.\mathrm{CDCl}_{3}\right): \delta 154.2,144.0,136.4,134.2,132.7,131.8,129.5,129.4,128.8,128.4,125.8$, 64.0, 38.6, 33.6, 27.3, 21.6 ppm; IR (KBr, $\left.\mathrm{cm}^{-1}\right)$ : 3067, 2922, 2851, 1598, 1095, 813, 694, 634, 583, 555, 538; HRMS (ESI) calcd for $\mathrm{C}_{20} \mathrm{H}_{21} \mathrm{~N}_{2} \mathrm{O}_{2} \mathrm{~S}^{+}(\mathrm{M}+\mathrm{H})^{+}$353.1318, found 353.1313 .

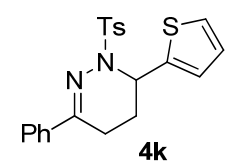

$48.8 \mathrm{mg}$, yield: 62\%; ${ }^{1} \mathrm{H}$ NMR (400 MHz, $\left.\mathrm{CDCl}_{3}\right): \delta 7.74-7.72(\mathrm{~m}, 2 \mathrm{H}), 7.61(\mathrm{~d}, J=$ $8.3 \mathrm{~Hz}, 2 \mathrm{H}), 7.39-7.36(\mathrm{~m}, 3 \mathrm{H}), 7.12(\mathrm{~d}, J=8.1 \mathrm{~Hz}, 2 \mathrm{H}), 7.06-7.05(\mathrm{~m}, 1 \mathrm{H})$, 6.79-6.77 (m, 1H), 6.75-6.74 (m, 1H), $5.86(\mathrm{t}, J=3.5 \mathrm{~Hz}, 1 \mathrm{H}), 2.72-2.65(\mathrm{~m}, 1 \mathrm{H})$, 2.52-2.45 (m, 1H), 2.34 (s, 3H), 2.23-2.19 (m, 2H) ppm; $\left.{ }^{13} \mathrm{C} \mathrm{NMR} \mathrm{(100} \mathrm{MHz,} \mathrm{CDCl}_{3}\right)$ : $\delta 148.1,143.4,141.8,136.9,135.4,129.3,129.0,128.4,128.0,126.3,126.1,125.4$, 124.8, 51.1, 25.4, 21.6, 18.5 ppm; IR (KBr, cm $\left.{ }^{-1}\right): 3065,2924,2854,1597,1446,937$, 
891, 757, 693, 670, 561; HRMS (ESI) calcd for $\mathrm{C}_{21} \mathrm{H}_{21} \mathrm{~N}_{2} \mathrm{O}_{2} \mathrm{~S}_{2}{ }^{+}(\mathrm{M}+\mathrm{H})^{+}$397.1039, found 397.1034 .

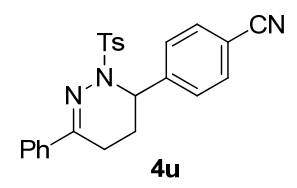

The compound $4 \mathrm{u}$ was used, when a Hammett plot was constructed to probe the electronic effects of the substrate alkene on the reaction rate (see, Figure 1D). $43.8 \mathrm{mg}$, yield: 53\%; ${ }^{1} \mathrm{H}$ NMR (400 MHz, $\left.\mathrm{CDCl}_{3}\right): \delta 7.73-7.71(\mathrm{~m}, 4 \mathrm{H}), 7.50(\mathrm{~d}, J=8.2 \mathrm{~Hz}$, 2H), 7.38-7.37 (m, 3H), $7.23(\mathrm{~d}, J=8.1 \mathrm{~Hz}, 2 \mathrm{H}), 7.15(\mathrm{~d}, J=8.2 \mathrm{~Hz}, 2 \mathrm{H}), 5.67(\mathrm{~s}$, $1 \mathrm{H}), 2.62(\mathrm{~d}, J=17.4 \mathrm{~Hz}, 1 \mathrm{H}), 2.40(\mathrm{~s}, 3 \mathrm{H}), 2.20-1.95(\mathrm{~m}, 3 \mathrm{H}) \mathrm{ppm} ;{ }^{13} \mathrm{C}$ NMR $(100$ $\left.\mathrm{MHz}, \mathrm{CDCl}_{3}\right): \delta 147.5,145.8,144.1,136.6,135.6,132.4,129.5,129.4,128.5,128.0$, 126.9, 125.3, 118.5, 111.4, 54.8, 24.5, 21.6, 17.7 ppm; IR ( $\left.\mathrm{KBr}, \mathrm{cm}^{-1}\right): 3065,2928$, 2856, 2228, 1608, 1447, 987, 940, 844, 757, 694; HRMS (ESI) calcd for $\mathrm{C}_{24} \mathrm{H}_{22} \mathrm{~N}_{3} \mathrm{O}_{2} \mathrm{~S}^{+}(\mathrm{M}+\mathrm{H})^{+} 416.1427$, found 416.1435 .

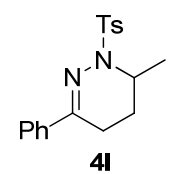

In a $30 \mathrm{ml}$ stainless steel reactor with a magnetic stirring bar was charged with $\mathrm{Cu}(\mathrm{MeCN})_{4} \mathrm{BF}_{4}(5 \mathrm{~mol} \%, 0.01 \mathrm{mmol})$ and diphenyl phosphate $1(5 \mathrm{~mol} \%, 0.01 \mathrm{mmol})$ in $0.5 \mathrm{~mL} \mathrm{CH}_{3} \mathrm{CN}$, stirred for $0.5 \mathrm{~h}$. Then, sulfonyl hydrazone $2 \mathrm{a}(0.2 \mathrm{mmol}$ in $0.4 \mathrm{~mL}$ $\left.\mathrm{CH}_{3} \mathrm{CN}\right), \mathrm{I}_{2}\left(5 \mathrm{~mol} \%, 2.54 \mathrm{mg}\right.$ in $\left.0.1 \mathrm{~mL} \mathrm{CH}_{3} \mathrm{CN}\right)$, TBHP $(0.8 \mathrm{mmol}$ in decane) were added successively to the reaction mixture. Next, the reaction mixture was charged with 8 atm propene $3 \mathbf{l}$ atmosphere and stirred at room temperature for $24 \mathrm{~h}$. After carefully releasing the propene, the mixture was evaporated and loaded on a silica column to give the final product 4l. $41.3 \mathrm{mg}$, yield: $63 \%$; ${ }^{1} \mathrm{H}$ NMR (400 $\left.\mathrm{MHz}, \mathrm{CDCl}_{3}\right)$ : $\delta 7.89(\mathrm{~d}, J=8.2 \mathrm{~Hz}, 2 \mathrm{H}), 7.73-7.70(\mathrm{~m}, 2 \mathrm{H}), 7.38-7.35(\mathrm{~m}, 3 \mathrm{H}), 7.28(\mathrm{~d}, J=8.2 \mathrm{~Hz}$, 2H), 4.61-4.58 (m, 1H), 2.65-2.59 (m, 1H), 2.48-2.41 (m, 1H), 2.39 (s, 3H), 1.91-1.88 $(\mathrm{m}, 2 \mathrm{H}), 1.15(\mathrm{~d}, J=6.6 \mathrm{~Hz}, 3 \mathrm{H}) \mathrm{ppm} ;{ }^{13} \mathrm{C} \mathrm{NMR}\left(100 \mathrm{MHz}, \mathrm{CDCl}_{3}\right): \delta 147.2,143.5$, 
137.0, 136.3, 129.4, 129.1, 128.3, 128.1, 125.2, 47.3, 24.3, 21.6, 18.2, 17.4 ppm; IR $\left(\mathrm{KBr}, \mathrm{cm}^{-1}\right): 3065,2930,2870,1597,1446,1234,912,836,762,694,592$; HRMS (ESI) calcd for $\mathrm{C}_{18} \mathrm{H}_{21} \mathrm{~N}_{2} \mathrm{O}_{2} \mathrm{~S}^{+}(\mathrm{M}+\mathrm{H})^{+} 329.1318$, found 329.1314 . 


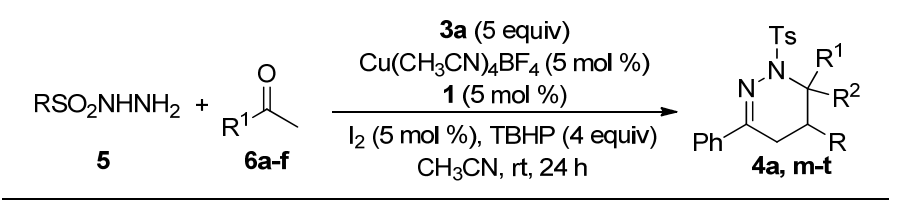

General procedure for one-pot oxidative [4+2] cycloaddition of sulfonyl

hydrazides 5 and ketones 6 with simple olefins 3: To an oven-dried reaction tube was added $\mathrm{Cu}\left(\mathrm{CH}_{3} \mathrm{CN}\right)_{4} \mathrm{BF}_{4}(0.01 \mathrm{mmol}, 5 \mathrm{~mol} \%)$ and diphenyl phosphate $1(0.01$ mmol, $5 \mathrm{~mol} \%)$. Distilled anhydrous $\mathrm{CH}_{3} \mathrm{CN}(0.5 \mathrm{~mL})$ was added. After stirring for $0.5 \mathrm{~h}$, sulfronyl hydrazide $5\left(0.2 \mathrm{mmol}\right.$, in $\left.0.2 \mathrm{~mL} \mathrm{CH}_{3} \mathrm{CN}\right)$, ketone $6(0.22 \mathrm{mmol}$, in $0.2 \mathrm{~mL} \mathrm{CH}_{3} \mathrm{CN}$ ) were added to the reaction mixture. After stirring for $0.5 \mathrm{~h}$, simple olefin 3 (1.0 mmol), $\mathrm{I}_{2}\left(5 \mathrm{~mol} \%\right.$, in $\left.0.1 \mathrm{~mL} \mathrm{CH}_{3} \mathrm{CN}\right)$, and TBHP $(0.8 \mathrm{mmol}$ in decane $)$ were added successively to the stirring reaction mixture. The mixture was stirred for 24-48 hours at room temperature. Purification of mixture by column chromatography on silica gel (petroleum ether:ethyl acetate $=20: 1$ to 10:1 as eluent) gave $\mathbf{4 a}, \mathbf{m}-\mathbf{t}$.

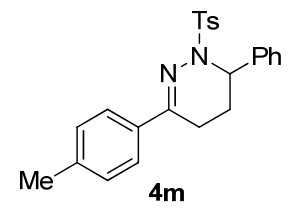

$54.8 \mathrm{mg}$, yield: $68 \%$; ${ }^{1} \mathrm{H}$ NMR $\left(400 \mathrm{MHz}, \mathrm{CDCl}_{3}\right): \delta$ 7.68-7.62 (m, 4H), 7.18-7.12 (m, 7H), $6.96(\mathrm{~d}, J=6.5 \mathrm{~Hz}, 2 \mathrm{H}), 5.63(\mathrm{~s}, 1 \mathrm{H}), 2.60-2.51(\mathrm{~m}, 1 \mathrm{H}), 2.36(\mathrm{~s}, 6 \mathrm{H}), 2.15-2.05$ (m, 3H) ppm; ${ }^{13} \mathrm{C}$ NMR (100 MHz, $\left.\mathrm{CDCl}_{3}\right): \delta$ 147.4, 143.4, 140.3, 139.3, 136.0, $134.3,129.1,129.1,128.4,128.1,127.3,126.1,125.2,55.2,24.9,21.5,21.3,17.8$ ppm; IR (KBr, cm $\left.{ }^{-1}\right): 3062,3029,2924,2862,1598,1495,1451,938,895,815,749$, 702, 548; HRMS (ESI) calcd for $\mathrm{C}_{24} \mathrm{H}_{25} \mathrm{~N}_{2} \mathrm{O}_{2} \mathrm{~S}^{+}(\mathrm{M}+\mathrm{H})^{+}$405.1631, found 405.1626.

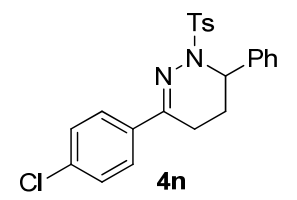

$55.3 \mathrm{mg}$, yield: $65 \% ;{ }^{1} \mathrm{H}$ NMR $\left(400 \mathrm{MHz}, \mathrm{CDCl}_{3}\right): \delta$ 7.67-7.64 (m, 4H), $7.33(\mathrm{~d}, J=$ 8.6 Hz, 2H), 7.20-7.14 (m, 5H), 6.96-6.94 (m, 2H), $5.64(\mathrm{~d}, J=2.8 \mathrm{~Hz}, 1 \mathrm{H})$, 2.57-2.46 (m, 1H), 2.37 (s, 3H), 2.18-2.01 (m, 3H) ppm; ${ }^{13} \mathrm{C} \mathrm{NMR}\left(100 \mathrm{MHz}, \mathrm{CDCl}_{3}\right)$ : $\delta 146.1,143.7,140.2,135.9,135.5,135.1,129.2,128.6,128.5,128.0,127.4,126.5$, 126.0, 55.2, 24.8, 21.6, 17.8 ppm; IR $\left(\mathrm{KBr}, \mathrm{cm}^{-1}\right): 3065,2926,2856,1600,1493$, 
1356, 1012, 939, 895, 814, 707, 664, 569; HRMS (ESI) calcd for $\mathrm{C}_{23} \mathrm{H}_{22} \mathrm{ClN}_{2} \mathrm{O}_{2} \mathrm{~S}^{+}$ $(\mathrm{M}+\mathrm{H})^{+}$425.1085, found 425.1079.

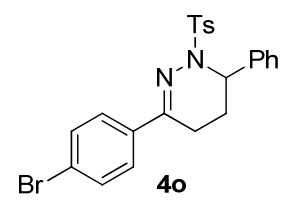

$56.8 \mathrm{mg}$, yield: $61 \% ;{ }^{1} \mathrm{H}$ NMR $\left(400 \mathrm{MHz}, \mathrm{CDCl}_{3}\right): \delta 7.66(\mathrm{~d}, J=8.2 \mathrm{~Hz}, 2 \mathrm{H}), 7.59$ (d, $J=8.6 \mathrm{~Hz}, 2 \mathrm{H}), 7.49$ (d, $J=8.6 \mathrm{~Hz}, 2 \mathrm{H}), 7.20-7.15(\mathrm{~m}, 5 \mathrm{H}), 6.96-6.94(\mathrm{~m}, 2 \mathrm{H}), 5.65$ $(\mathrm{d}, J=3.2 \mathrm{~Hz}, 1 \mathrm{H}), 2.57-2.48(\mathrm{~m}, 1 \mathrm{H}), 2.38(\mathrm{~s}, 3 \mathrm{H}), 2.18-2.06(\mathrm{~m}, 3 \mathrm{H}) \mathrm{ppm} ;{ }^{13} \mathrm{C}$ NMR (100 MHz, $\left.\mathrm{CDCl}_{3}\right): \delta 146.1,143.7,140.1,135.9,135.9,131.5,129.2,128.5$, 128.1, 127.4, 126.8, 126.0, 123.4, 55.2, 24.8, 21.6, 17.7 ppm; IR $\left(\mathrm{KBr}, \mathrm{cm}^{-1}\right): 3065$, 2926, 2856, 1599, 1490, 1007, 939, 895, 787, 688, 661, 564; HRMS (ESI) calcd for $\mathrm{C}_{23} \mathrm{H}_{22} \mathrm{BrN}_{2} \mathrm{O}_{2} \mathrm{~S}^{+}(\mathrm{M}+\mathrm{H})^{+} 469.0580$, found 469.0573 .

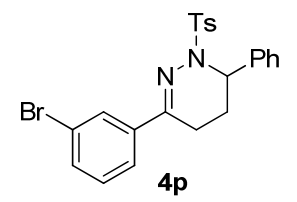

$54.2 \mathrm{mg}$, yield: $58 \%$; ${ }^{1} \mathrm{H}$ NMR $\left(400 \mathrm{MHz}, \mathrm{CDCl}_{3}\right): \delta 7.84(\mathrm{~s}, 1 \mathrm{H}), 7.68(\mathrm{~d}, J=8.2 \mathrm{~Hz}$, 2H), $7.63(\mathrm{~d}, J=6.9 \mathrm{~Hz}, 1 \mathrm{H}), 7.46(\mathrm{~d}, J=8.0 \mathrm{~Hz}, 1 \mathrm{H}), 7.25-7.15(\mathrm{~m}, 6 \mathrm{H}), 6.96-6.94$ (m, 2H), $5.65(\mathrm{~d}, J=3.3 \mathrm{~Hz}, 1 \mathrm{H}), 2.57-2.47(\mathrm{~m}, 1 \mathrm{H}), 2.38(\mathrm{~s}, 3 \mathrm{H}), 2.18-2.05(\mathrm{~m}, 3 \mathrm{H})$ ppm; ${ }^{13} \mathrm{C}$ NMR (100 MHz, $\left.\mathrm{CDCl}_{3}\right): \delta 145.7,143.7,140.1,139.1,135.8,131.9,129.9$, $129.3,128.5,128.4,128.1,127.4,126.0,123.8,122.7,55.2,24.8,21.6,17.9$ ppm; IR $\left(\mathrm{KBr}, \mathrm{cm}^{-1}\right):$ 3062, 2929, 2854, 1597, 1451, 1101, 995, 940, 894, 801, 697, 589; HRMS (ESI) calcd for $\mathrm{C}_{23} \mathrm{H}_{22} \mathrm{BrN}_{2} \mathrm{O}_{2} \mathrm{~S}^{+}(\mathrm{M}+\mathrm{H})^{+}$469.0580, found 469.0573.

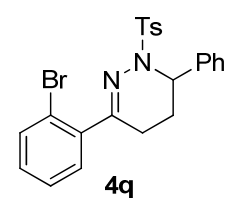

$64.4 \mathrm{mg}$, yield: 69\%; ${ }^{1} \mathrm{H}$ NMR (400 MHz, $\left.\mathrm{CDCl}_{3}\right): \delta 7.70(\mathrm{~d}, J=8.2 \mathrm{~Hz}, 2 \mathrm{H}), 7.53(\mathrm{~d}$, $J=8.0 \mathrm{~Hz}, 1 \mathrm{H}), 7.32-7.13(\mathrm{~m}, 10 \mathrm{H}), 5.60(\mathrm{~d}, J=1.4 \mathrm{~Hz}, 1 \mathrm{H}), 2.41(\mathrm{~s}, 3 \mathrm{H}), 2.37-2.31$ $(\mathrm{m}, 1 \mathrm{H}), 2.26-2.17(\mathrm{~m}, 1 \mathrm{H}), 2.11-2.06(\mathrm{~m}, 1 \mathrm{H}), 1.97-1.89(\mathrm{~m}, 1 \mathrm{H}) \mathrm{ppm} ;{ }^{13} \mathrm{C} \mathrm{NMR}$ $\left(100 \mathrm{MHz}, \mathrm{CDCl}_{3}\right): \delta 151.1,143.6,140.7,140.0,136.0,133.1,130.3,129.9,129.3$, $128.5,128.0,127.4,127.4,126.1,121.5,55.5,24.3,21.9,21.6$ ppm; IR $\left(\mathrm{KBr}, \mathrm{cm}^{-1}\right)$ : 
3062, 2926, 2859, 1597, 1494, 1451, 987, 936, 891, 789, 703, 584; HRMS (ESI) calcd for $\mathrm{C}_{23} \mathrm{H}_{22} \mathrm{BrN}_{2} \mathrm{O}_{2} \mathrm{~S}^{+}(\mathrm{M}+\mathrm{H})^{+}$469.0580, found 469.0574.

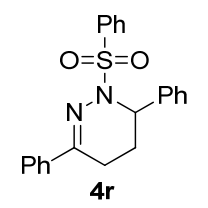

$54.2 \mathrm{mg}$, yield: $72 \%$; ${ }^{1} \mathrm{H}$ NMR $\left(400 \mathrm{MHz}, \mathrm{CDCl}_{3}\right): \delta$ 7.81-7.78 (m, 2H), 7.75-7.73 (m, 2H), $7.50(\mathrm{t}, J=7.5 \mathrm{~Hz}, 1 \mathrm{H}), 7.39-7.36(\mathrm{~m}, 5 \mathrm{H}), 7.19-7.13(\mathrm{~m}, 3 \mathrm{H}), 6.95(\mathrm{~d}, J=6.6$ $\mathrm{Hz}, 2 \mathrm{HHHH}), 5.66$ (d, $J=2.3 \mathrm{~Hz}, 1 \mathrm{H}), 2.64-2.56(\mathrm{~m}, 1 \mathrm{H}), 2.18-2.11$ (m, 3H) ppm; ${ }^{13} \mathrm{C} \mathrm{NMR}\left(100 \mathrm{MHz} \mathrm{CDCl}_{3}\right): \delta 147.5,140.1,138.9,137.0,132.6,129.2,128.5,128.5$, 128.4, 128.0, 127.3, 126.0, 125.3, 55.2, 24.9, 17.9 ppm; IR (KBr, cm $\left.{ }^{-1}\right): 3065,2924$, 2854, 1447, 939, 894, 792, 754, 721, 689, 654, 555; HRMS (ESI) calcd for $\mathrm{C}_{22} \mathrm{H}_{21} \mathrm{~N}_{2} \mathrm{O}_{2} \mathrm{~S}^{+}(\mathrm{M}+\mathrm{H})^{+}$377.1318, found 377.1314.

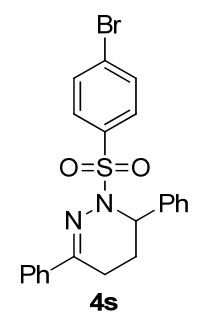

$45.3 \mathrm{mg}$, yield: 50\%; ${ }^{1} \mathrm{H}$ NMR (400 MHz, $\left.\mathrm{CDCl}_{3}\right): \delta$ 7.74-7.72 (m, 2H), $7.61(\mathrm{~d}, J=$ $8.5 \mathrm{~Hz}, 2 \mathrm{H}), 7.49$ (d, $J=8.5 \mathrm{~Hz}, 2 \mathrm{H}), 7.39-7.38(\mathrm{~m}, 3 \mathrm{H}), 7.21-7.14(\mathrm{~m}, 3 \mathrm{H}), 6.92(\mathrm{~d}, J$ $=7.0 \mathrm{~Hz}, 2 \mathrm{HHHH}), 5.63(\mathrm{~s}, 1 \mathrm{H}), 2.68-2.58(\mathrm{~m}, 1 \mathrm{H}), 2.24-2.14(\mathrm{~m}, 3 \mathrm{H}) \mathrm{ppm} ;{ }^{13} \mathrm{C}$ NMR (100 MHz, $\left.\mathrm{CDCl}_{3}\right): \delta 148.0,139.9,137.8,136.8,131.7,129.6,129.4,128.6$, 128.5, 127.7, 127.5, 126.1, 125.3, 55.3, 25.0, 17.9 ppm; IR $\left(\mathrm{KBr}, \mathrm{cm}^{-1}\right)$ : 3065, 2923, 2854, 1574, 1361, 1094, 792, 739, 700, 599, 554; HRMS (ESI) calcd for $\mathrm{C}_{22} \mathrm{H}_{20} \mathrm{BrN}_{2} \mathrm{O}_{2} \mathrm{~S}^{+}(\mathrm{M}+\mathrm{H})^{+} 455.0423$, found 455.0417 .

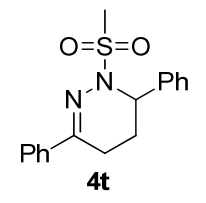

$29.0 \mathrm{mg}$, yield: 46\%; ${ }^{1} \mathrm{H}$ NMR (400 MHz, $\left.\mathrm{CDCl}_{3}\right): \delta$ 7.81-7.78 (m, 2H), 7.43-7.39 (m, 3H), 7.34-7.26 (m, 3H), $7.19(\mathrm{~d}, J=7.3 \mathrm{~Hz}, 2 \mathrm{H}), 5.60(\mathrm{~d}, J=3.1 \mathrm{~Hz}, 1 \mathrm{H}), 3.08(\mathrm{~s}$, $3 \mathrm{H}), 2.76-2.65(\mathrm{~m}, 1 \mathrm{H}), 2.32-2.22(\mathrm{~m}, 3 \mathrm{H}) \mathrm{ppm} ;{ }^{13} \mathrm{C} \mathrm{NMR}\left(100 \mathrm{MHz}, \mathrm{CDCl}_{3}\right): \delta$ $147.5,140.8,136.8,129.4,128.8,128.5,127.7,125.9,125.4,54.4,39.3,25.1,18.1$ 
ppm; IR (KBr, cm $\left.{ }^{-1}\right): 3062,3029,2929,1495,1448,1242,1069,939,894,796,695$; HRMS (ESI) calcd for $\mathrm{C}_{17} \mathrm{H}_{19} \mathrm{~N}_{2} \mathrm{O}_{2} \mathrm{~S}^{+}(\mathrm{M}+\mathrm{H})^{+} 315.1162$, found 315.1159. 


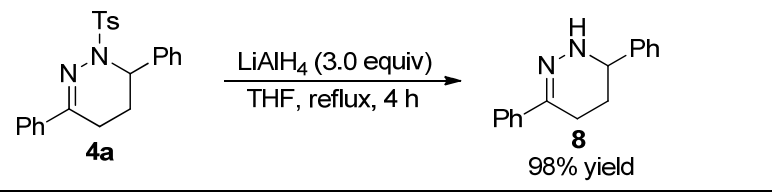

Transformation of Product 4a: To $\mathrm{LiAlH}_{4}(3.0$ equiv, $22.8 \mathrm{mg}, 0.6 \mathrm{mmol}$ ) solution in THF $(2 \mathrm{~mL})$ was added tetrahydropyridazine $4 \mathrm{a}(78.0 \mathrm{mg}, 0.2 \mathrm{mmol})$ at room temperature. After refluxing for $4 \mathrm{~h}$, the reaction mixture was quenched by cold water, then extracted with EtOAc $(3 \times 10 \mathrm{~mL})$. The combined organic layers were dried $\left(\mathrm{Na}_{2} \mathrm{SO}_{4}\right)$, evaporated under reduced pressure and purified by flash chromatography on silica gel (petroleum ether:ethyl acetate $=10: 1$ as eluent) to afford the desired product 8 as a white solid.

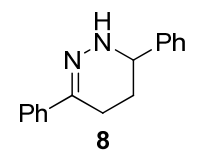

$46.5 \mathrm{mg}$, yield: 98\%; ${ }^{1} \mathrm{H} \mathrm{NMR}\left(400 \mathrm{MHz}, \mathrm{CDCl}_{3}\right): \delta 7.68(\mathrm{~d}, J=7.8 \mathrm{~Hz}, 2 \mathrm{H})$, 7.41-7.27 (m, 8H), $5.81(\mathrm{br}, 1 \mathrm{H}), 4.17-4.14(\mathrm{~m}, 1 \mathrm{H}), 2.84-2.75(\mathrm{~m}, 1 \mathrm{H}), 2.71-2.64(\mathrm{~m}$, 1H), 2.26-2.13 (m, 2H) ppm; ${ }^{13} \mathrm{C}$ NMR (100 MHz, $\left.\mathrm{CDCl}_{3}\right): \delta 143.2,142.1,138.6$, 128.7, 128.3, 127.9, 127.8, 127.0, 124.5, 56.4, 28.6, 23.4 ppm; IR $\left(\mathrm{KBr}, \mathrm{cm}^{-1}\right): 3055$, 3031, 2926, 2854, 1681, 1581, 1494, 1408, 1126, 867, 589; HRMS (ESI) calcd for $\mathrm{C}_{16} \mathrm{H}_{17} \mathrm{~N}_{2}^{+}(\mathrm{M}+\mathrm{H})^{+} 237.1386$, found 237.1383. 


\section{Mechanistic Studies of Binary-acid Catalysis}

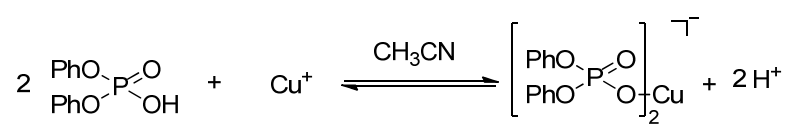

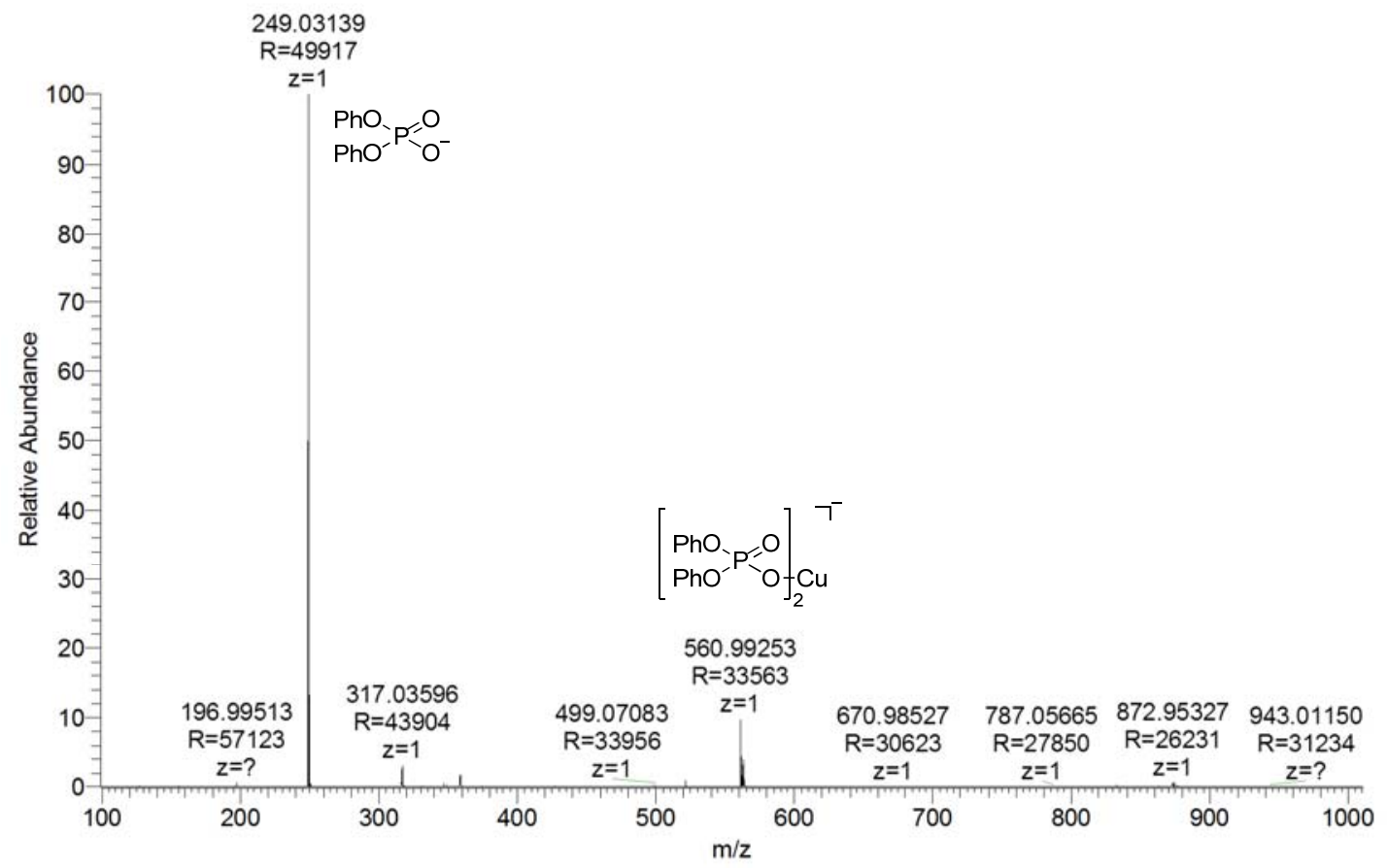

Figure S1. ESI-MS Analysis of Binary acid $\mathrm{Cu}\left(\mathrm{CH}_{3} \mathrm{CN}\right)_{4} \mathrm{BF}_{4} / \mathbf{1}$. 
(B)

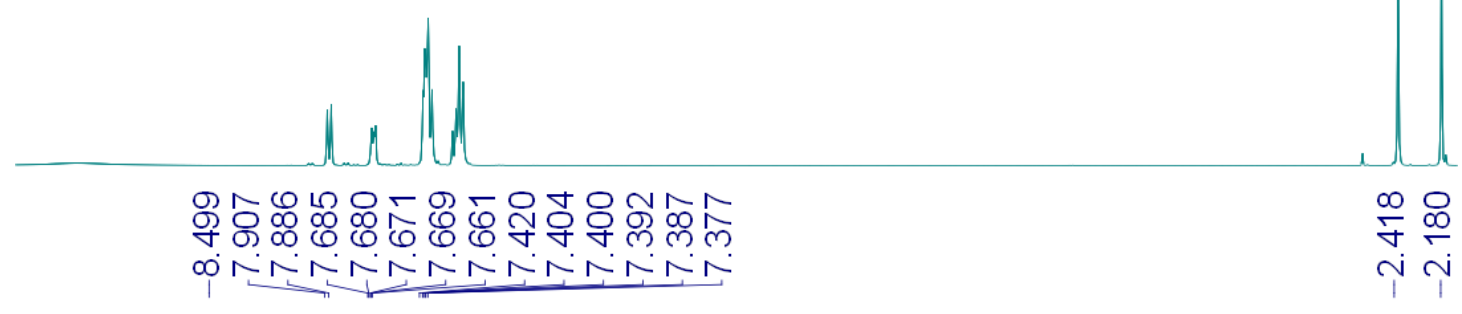

(A)

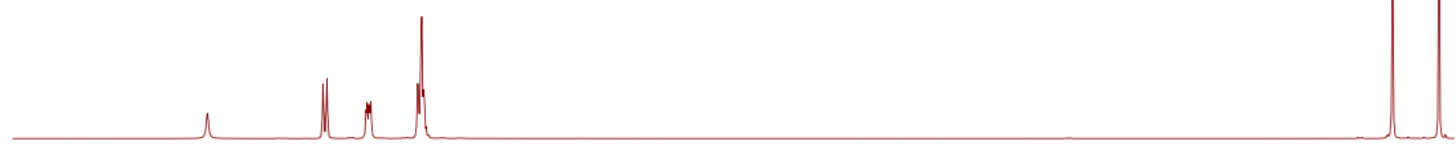

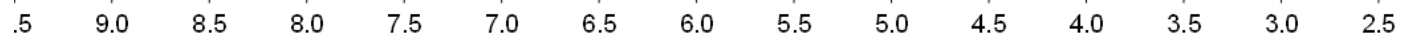

Figure S2. (A) ${ }^{1} \mathrm{H}$ NMR spectrum of $N$-tosylhydrazone 2a; (B) ${ }^{1} \mathrm{H}$ NMR spectrum of the complexes of $N$-tosylhydrazone $2 \mathrm{a}, \mathrm{Cu}\left(\mathrm{CH}_{3} \mathrm{CN}\right)_{4} \mathrm{BF}_{4}$ and diphenyl phosphate $\mathbf{1}$ $(1: 1: 1)$.

Weak but noticeable shift of the hydrazine signals were observed, in particular, shift of hydrazine N-H was clearly noted. 
Table S2. Results of different iodine source ${ }^{[\mathrm{a}]}$

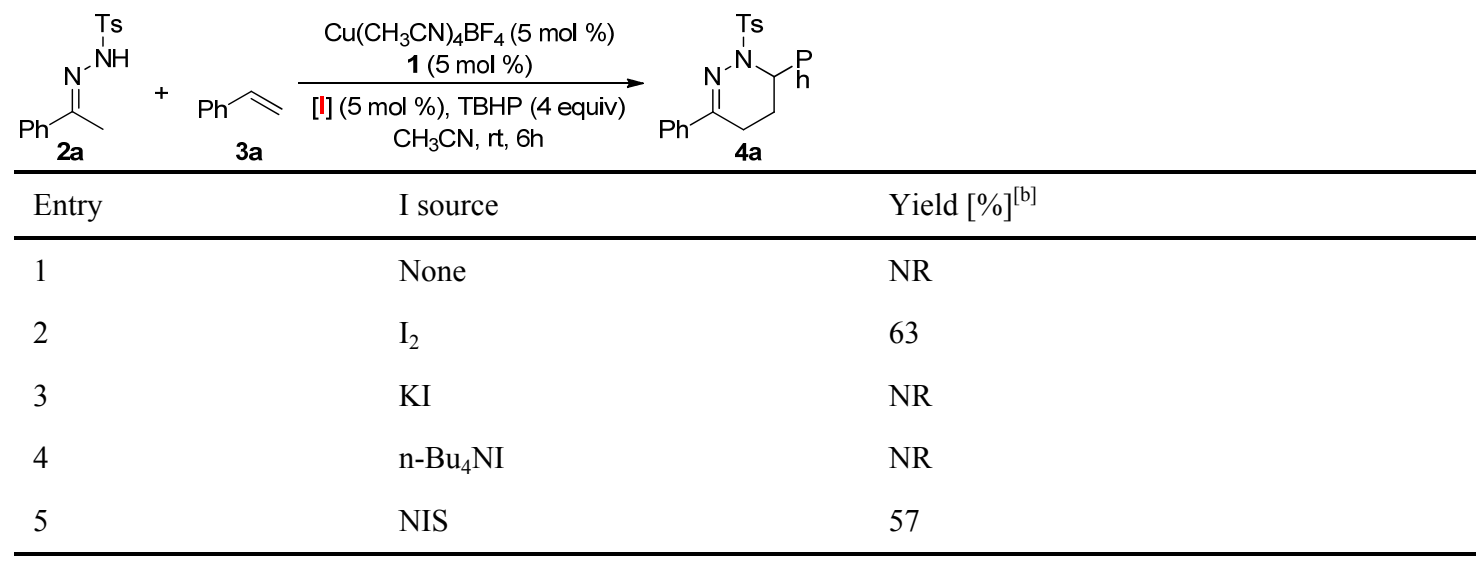

[a] Standard reaction condition: $\mathrm{Cu}\left(\mathrm{CH}_{3} \mathrm{CN}\right)_{4} \mathrm{BF}_{4}(5 \mathrm{~mol} \%), 1(5 \mathrm{~mol} \%)$, I source $(5 \mathrm{~mol} \%)$, TBHP $(0.8 \mathrm{mmol})$, 2a $(0.2 \mathrm{mmol})$, and $3 \mathrm{a}(1.0 \mathrm{mmol})$ at room temperature in $\mathrm{CH}_{3} \mathrm{CN}$ for $6 \mathrm{~h}$. [b] Determined by ${ }^{1} \mathrm{H}$ NMR analysis with an internal standard, 1,3,5-trimethyloxylbenzene. 
(A)
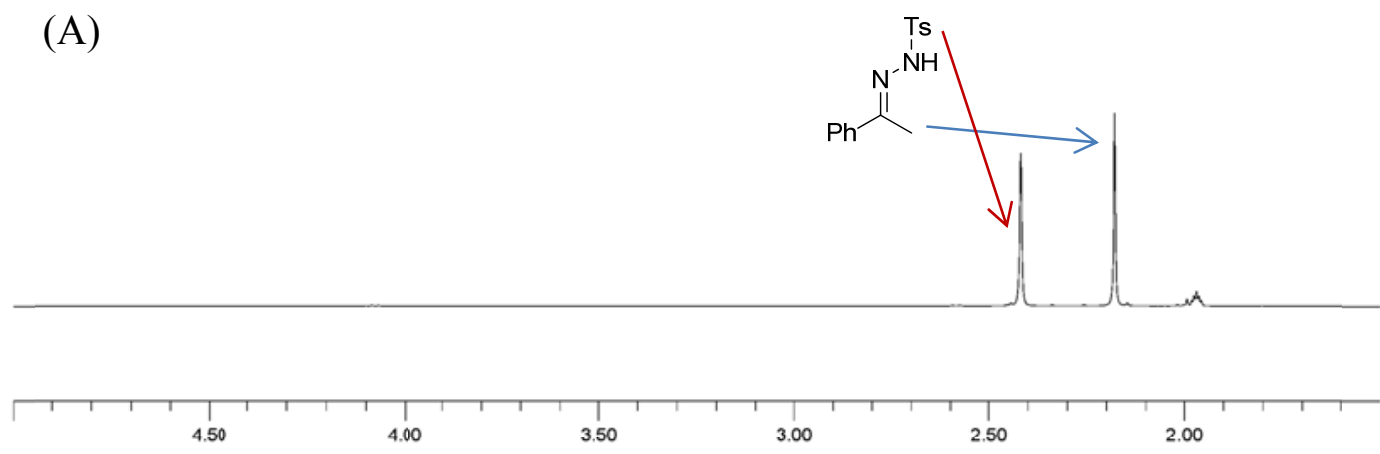

(B)
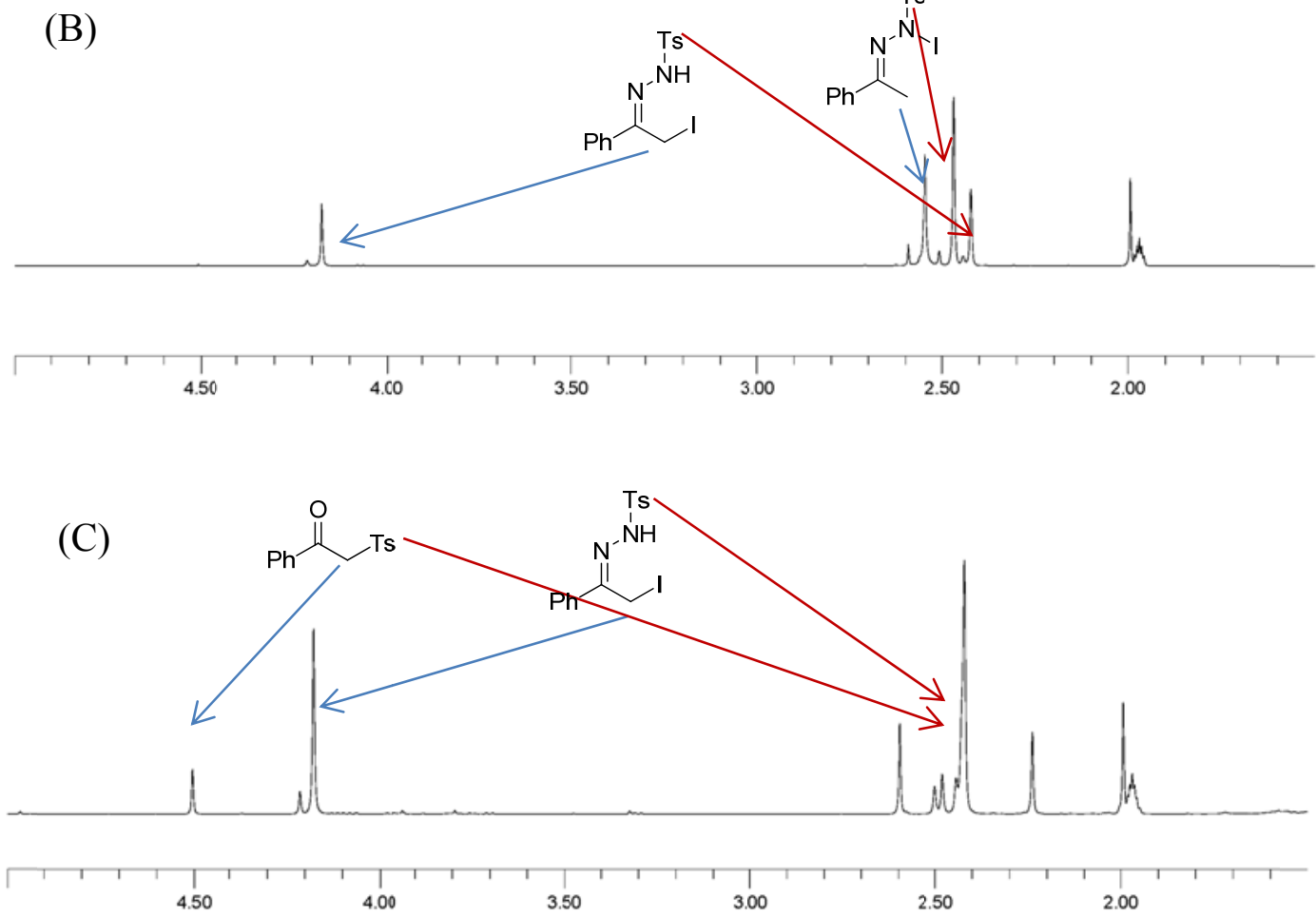

(D)

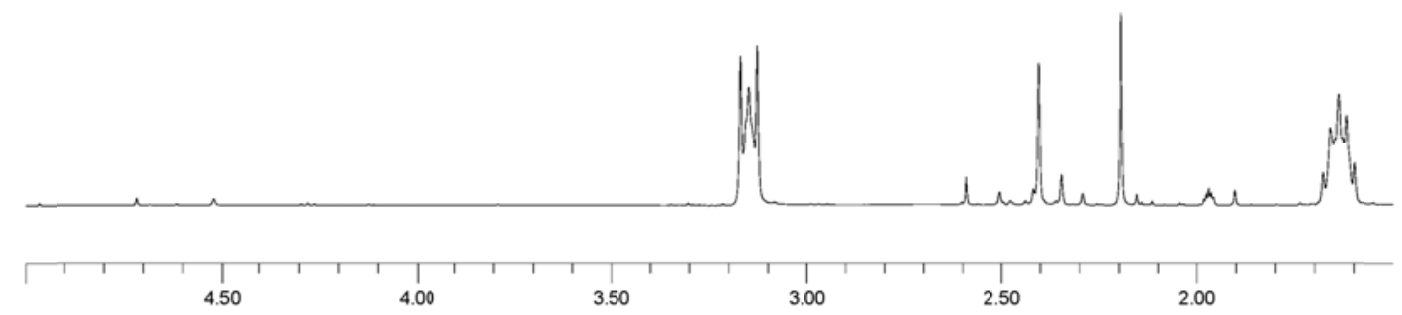

Figure S3. Reaction conditions: (A) $2 a(0.1 \mathrm{mmol})$ in $\mathrm{CD}_{3} \mathrm{CN}$; (B) $\mathrm{Cu}\left(\mathrm{CH}_{3} \mathrm{CN}\right)_{4} \mathrm{BF}_{4}$ (5 mol\%), 1 (5 mol\%), 2a $(0.1 \mathrm{mmol})$ and $\mathrm{I}_{2}(0.1 \mathrm{mmol})$ in $\mathrm{CD}_{3} \mathrm{CN}$, stirring at $1 \mathrm{~h} ;(\mathrm{C})$ Adding TBHP (4.0 equiv) in B' mixture; (D) $\mathrm{Cu}\left(\mathrm{CH}_{3} \mathrm{CN}\right)_{4} \mathrm{BF}_{4}(5 \mathrm{~mol} \%), 1$ (5 mol\%), 2a $(0.1 \mathrm{mmol}),(\mathrm{n}-\mathrm{Bu})_{4} \mathrm{NI}_{2}(0.1 \mathrm{mmol})$ and TBHP $\left(0.4\right.$ equiv) in $\mathrm{CD}_{3} \mathrm{CN}$ 


\section{Control experiment}

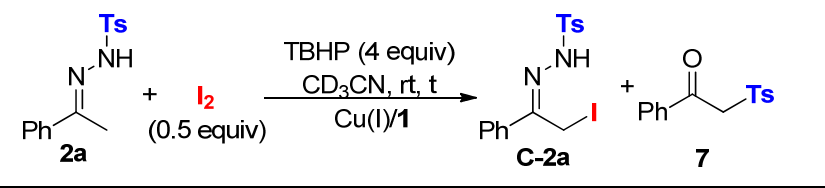

Procedure A: To an oven-dried reaction tube was added $\mathrm{Cu}\left(\mathrm{CH}_{3} \mathrm{CN}\right)_{4} \mathrm{BF}_{4}(5 \mathrm{~mol} \%)$ and diphenyl phosphate 1 (5 mol\%). Anhydrous $\mathrm{CD}_{3} \mathrm{CN}(0.3 \mathrm{~mL})$ was added. After stirring for $0.5 \mathrm{~h}$, sulfonyl hydrazine $2 \mathrm{a}(0.1 \mathrm{mmol}), \mathrm{I}_{2}\left(50 \mathrm{~mol} \%\right.$, in $\left.0.2 \mathrm{~mL} \mathrm{CD}_{3} \mathrm{CN}\right)$, and TBHP (0.4 mmol in decane) were added successively to the stirring reaction mixture. The mixture was stirred for 0.5-3 hours at room temperature. The reaction progress was monitored by ${ }^{1} \mathrm{H}$ NMR (Scheme 4B).

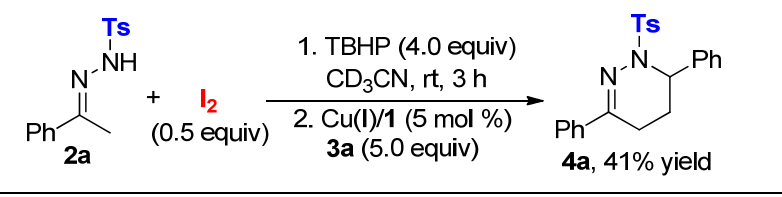

Procedure B: To an oven-dried reaction tube was added hydrazine $2 \mathrm{a}(0.1 \mathrm{mmol})$. Anhydrous $\mathrm{CH}_{3} \mathrm{CN}(0.2 \mathrm{~mL})$ was added. And then $\mathrm{I}_{2}\left(50 \mathrm{~mol} \%\right.$, in $\left.0.2 \mathrm{~mL} \mathrm{CH}_{3} \mathrm{CN}\right)$, and TBHP (0.4 mmol in decane) were added successively to the stirring reaction mixture. After stirring for 3 hours at room temperature, $\mathrm{Cu}\left(\mathrm{CH}_{3} \mathrm{CN}\right)_{4} \mathrm{BF}_{4}(5 \mathrm{~mol} \%)$ and diphenyl phosphate $1(5 \mathrm{~mol} \%$, in $0.1 \mathrm{~mL})$ was added to the mixture. Anhydrous $\mathrm{CD}_{3} \mathrm{CN}(0.4 \mathrm{~mL})$ was added. And then styrene $3 \mathbf{a}(0.5 \mathrm{mmol})$ was added. The reaction progress was monitored by ${ }^{1} \mathrm{H}$ NMR (Scheme $4 \mathrm{C}$ ), and yield was determined by ${ }^{1} \mathrm{H}$ NMR analysis with an internal standard (1,3,5-trimethyloxyl benzene). 
Kinetics study: iodination reaction

(A)

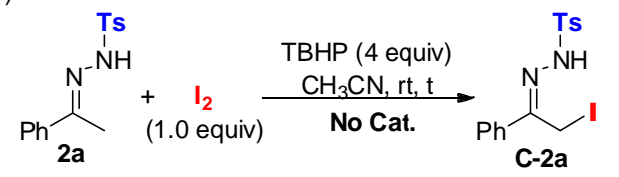

Procedure A for catalyst-free iodination reaction of sulfonyl hydrazine 2a, monitored by in-situ IR: To an oven-dried reaction tube was added hydrazine 2a ( 0.2 mmol). Anhydrous $\mathrm{CH}_{3} \mathrm{CN}(0.8 \mathrm{~mL})$ was added. And then $\mathrm{I}_{2}(100 \mathrm{~mol} \%$, in $0.2 \mathrm{~mL}$ $\left.\mathrm{CH}_{3} \mathrm{CN}\right)$, and TBHP (0.8 mmol in decane) were added successively to the stirring reaction mixture. At the same time, transformation of C-2a was also tracked by in situ IR in this reaction (see Figure 1A). The reaction progress was monitored by in-situ IR at $1269 \mathrm{~cm}^{-1}$ (Figure S4).

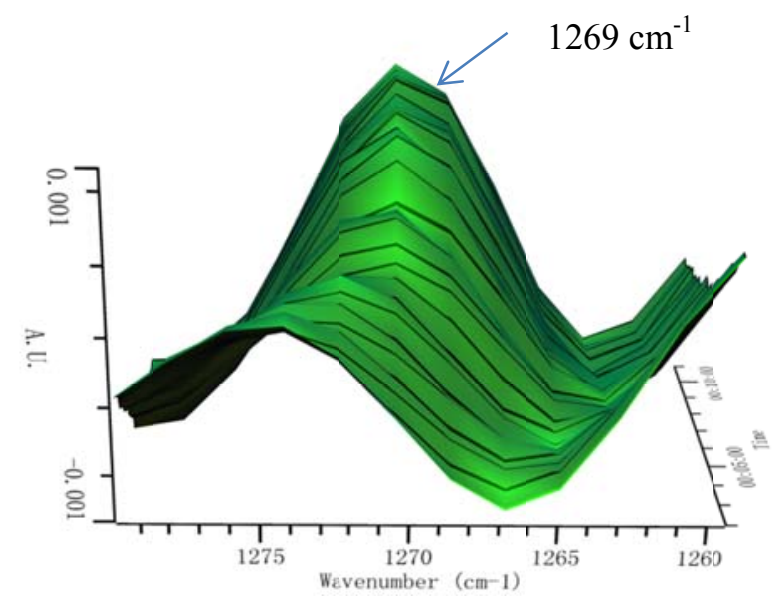

Figure S4. Representative 3D stacking plots of spectra obtained by React-IR

(B)

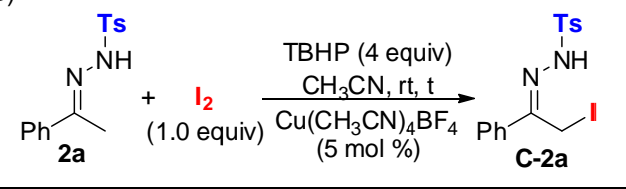

Procedure B for iodination reaction of sulfonyl hydrazine 2a catalyzed by $\underline{\mathrm{Cu}\left(\mathrm{CH}_{3}\right.} \underline{\mathrm{CN}}_{4} \underline{\mathrm{BF}}_{4}$, monitored by in-situ IR: To an oven-dried reaction tube was added $\mathrm{Cu}\left(\mathrm{CH}_{3} \mathrm{CN}\right)_{4} \mathrm{BF}_{4}(5 \mathrm{~mol} \%)$ and hydrazine $2 \mathrm{a}(0.2 \mathrm{mmol})$. Anhydrous $\mathrm{CH}_{3} \mathrm{CN}$ $(0.8 \mathrm{~mL})$ was added. And then $\mathrm{I}_{2}\left(100 \mathrm{~mol} \%\right.$, in $\left.0.2 \mathrm{~mL} \mathrm{CH}_{3} \mathrm{CN}\right)$, and TBHP $(0.8$ mmol in decane) were added successively to the stirring reaction mixture. At the same time, transformation of C-2a was also tracked by in situ IR in this reaction (see Figure 
1A). The reaction progress was monitored by in-situ IR at $1269 \mathrm{~cm}^{-1}$ (Figure S4).

(C)

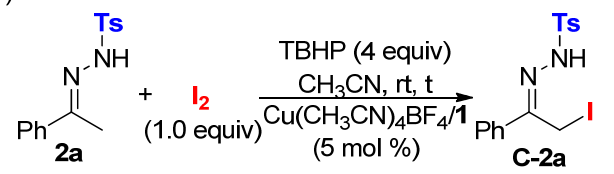

Procedure C for iodination reaction of sulfonyl hydrazine 2a catalyzed by binary-acids, monitored by in-situ IR: To an oven-dried reaction tube was added $\mathrm{Cu}\left(\mathrm{CH}_{3} \mathrm{CN}\right)_{4} \mathrm{BF}_{4}(0.01 \mathrm{mmol}, 5 \mathrm{~mol} \%)$ and diphenyl phosphate $1(0.01 \mathrm{mmol}, 5$ mol\%). Distilled anhydrous $\mathrm{CH}_{3} \mathrm{CN}(0.6 \mathrm{~mL})$ was added. After stirring for $0.5 \mathrm{~h}$, sulfonyl hydrazine 2a $\left(0.2 \mathrm{mmol}\right.$, in $\left.0.2 \mathrm{~mL} \mathrm{CH}{ }_{3} \mathrm{CN}\right), \mathrm{I}_{2}(100 \mathrm{~mol} \%$, in $0.2 \mathrm{~mL}$ $\left.\mathrm{CH}_{3} \mathrm{CN}\right)$, and TBHP (0.8 mmol in decane) were added successively to the stirring reaction mixture. At the same time, transformation of C-2a was also tracked by in situ IR in this reaction (see Figure 1A). The reaction progress was monitored by in-situ IR at $1269 \mathrm{~cm}^{-1}$ (Figure S4). 
Kinetics study: oxidative [4+2] cylcoaddition reaction

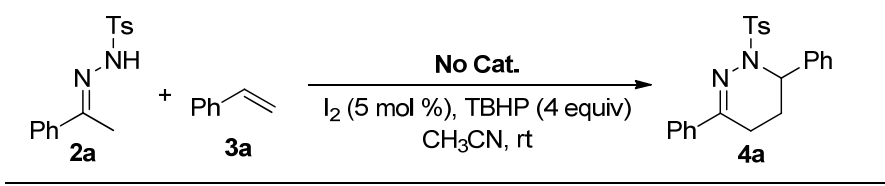

Procedure A for catalyst-free oxidative [4+2] reaction of sulfonyl hydrazine 2a and styrene 3a, monitored by in-situ IR: To an oven-dried reaction tube was added hydrazine 2a $(0.2 \mathrm{mmol})$. Anhydrous $\mathrm{CH}_{3} \mathrm{CN}(0.9 \mathrm{~mL})$ was added. And then styrene 3a (1.0 mmol), $\mathrm{I}_{2}\left(5 \mathrm{~mol} \%\right.$, in $\left.0.1 \mathrm{~mL} \mathrm{CH}_{3} \mathrm{CN}\right)$, and TBHP $(0.8 \mathrm{mmol}$ in decane $)$ were added successively to the stirring reaction mixture. At the same time, transformation of 4a was also tracked by in situ IR in this reaction (see Figure 1B). The reaction progress was monitored by in-situ IR at $895 \mathrm{~cm}^{-1}$ (Figure S5).

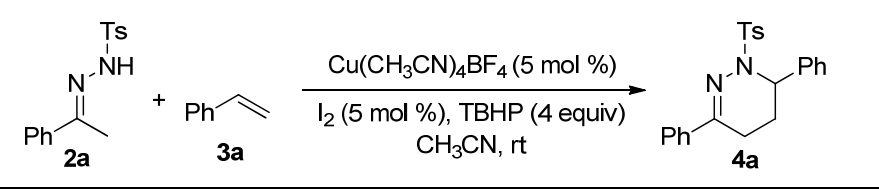

Procedure B for oxidative [4+2] reaction of sulfonyl hydrazine 2a and styrene 3a, $\underline{\text { catalyzed by } \mathrm{Cu}\left(\mathrm{CH}_{3}\right.} \underline{\mathrm{CN}}_{4} \underline{\mathrm{BF}}_{4}$, monitored by in-situ IR: To an oven-dried reaction tube was added $\mathrm{Cu}\left(\mathrm{CH}_{3} \mathrm{CN}\right)_{4} \mathrm{BF}_{4}(5 \mathrm{~mol} \%)$ and hydrazine 2a $(0.2 \mathrm{mmol})$. Anhydrous $\mathrm{CH}_{3} \mathrm{CN}(0.9 \mathrm{~mL})$ was added. And then styrene 3a, $\mathrm{I}_{2}\left(5 \mathrm{~mol} \%\right.$, in $\left.0.1 \mathrm{~mL} \mathrm{CH}_{3} \mathrm{CN}\right)$, and TBHP (0.8 mmol in decane) were added successively to the stirring reaction mixture. At the same time, transformation of $\mathbf{4 a}$ was also tracked by in situ IR in this reaction (see Figure 1B). The reaction progress was monitored by in-situ IR at 895 $\mathrm{cm}^{-1}$ (Figure S5).

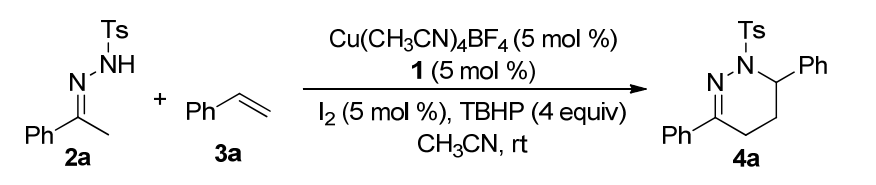

Procedure $C$ for oxidative [4+2] reaction of sulfonyl hydrazine 2a and styrene 3a, catalyzed by $\mathrm{Cu}\left(\mathrm{CH}_{3} \underline{\mathrm{CN}}\right)_{4} \underline{\mathrm{BF}}_{4} / \mathbf{1}$, monitored by in-situ IR: To an oven-dried reaction tube was added $\mathrm{Cu}\left(\mathrm{CH}_{3} \mathrm{CN}\right)_{4} \mathrm{BF}_{4}(0.01 \mathrm{mmol}, 5 \mathrm{~mol} \%)$ and diphenyl phosphate 1 (0.01 mmol, 5 mol\%). Distilled anhydrous $\mathrm{CH}_{3} \mathrm{CN}(0.8 \mathrm{~mL})$ was added. After stirring for $0.5 \mathrm{~h}$, sulfonyl hydrazine 2a $\left(0.2 \mathrm{mmol}\right.$, in $\left.0.1 \mathrm{~mL} \mathrm{CH}_{3} \mathrm{CN}\right)$, styrene 3a (1.0 mmol), $\mathrm{I}_{2}\left(5 \mathrm{~mol} \%\right.$, in $\left.0.1 \mathrm{~mL} \mathrm{CH}_{3} \mathrm{CN}\right)$, and TBHP $(0.8 \mathrm{mmol}$ in decane) were 
added successively to the stirring reaction mixture. At the same time, transformation of 4a was also tracked by in situ IR in this reaction (see Figure 1B). The reaction progress was monitored by in-situ IR at $895 \mathrm{~cm}^{-1}$ (Figure S5).

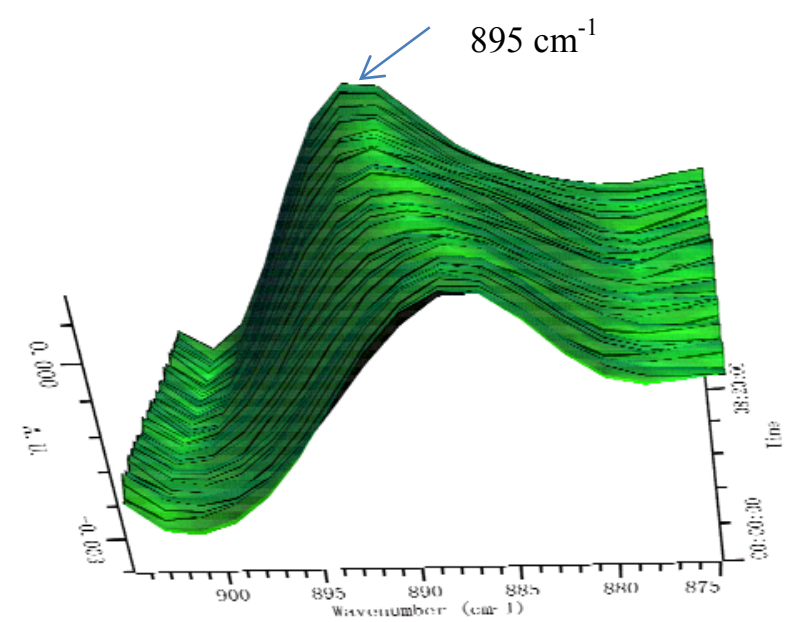

Figure S5. Representative 3D stacking plots of spectra obtained by React-IR

Procedure D for oxidative [4+2] reaction of sulfonyl hydrazine 2a and styrene 3a, catalyzed by $\mathrm{Cu}\left(\mathrm{CH}_{3} \underline{\mathrm{CN}}\right)_{4} \underline{\mathrm{BF}}_{4} / 1$, monitored by continuous ${ }^{1} \mathrm{H} N M R$ : To be added $\mathrm{Cu}\left(\mathrm{CH}_{3} \mathrm{CN}\right)_{4} \mathrm{BF}_{4}(0.005 \mathrm{mmol}, 5 \mathrm{~mol} \%)$ and diphenyl phosphate $1(0.005 \mathrm{mmol}, 5$ mol\%), anhydrous $\mathrm{CD}_{3} \mathrm{CN}(0.2 \mathrm{~mL})$ was added. After stirring for $0.5 \mathrm{~h}$, binary-acids, sulfonyl hydrazine 2a $\left(0.1 \mathrm{mmol}\right.$, in $\left.0.1 \mathrm{~mL} \mathrm{CD}{ }_{3} \mathrm{CN}\right)$, styrene $3 \mathbf{a}(1.0 \mathrm{mmol}), \mathrm{I}_{2}(50$ $\mathrm{mol} \%$, in $\left.0.4 \mathrm{~mL} \mathrm{CD}{ }_{3} \mathrm{CN}\right)$, and TBHP (0.4 mmol in decane) were added successively to the NMR tube. At the same time, transformation of 4a and C-2a was also tracked by continuous ${ }^{1} \mathrm{H}$ NMR in this reaction (see Figure $1 \mathrm{C}$ ). The reaction progress was monitored by continuous ${ }^{1} \mathrm{H}$ NMR at $4.13 \mathrm{ppm}$ (adduct C-2a generated) and $5.60 \mathrm{ppm}$ (adduct 4a) $\mathrm{cm}^{-1}$ (see some selected examples in Figure S6). 


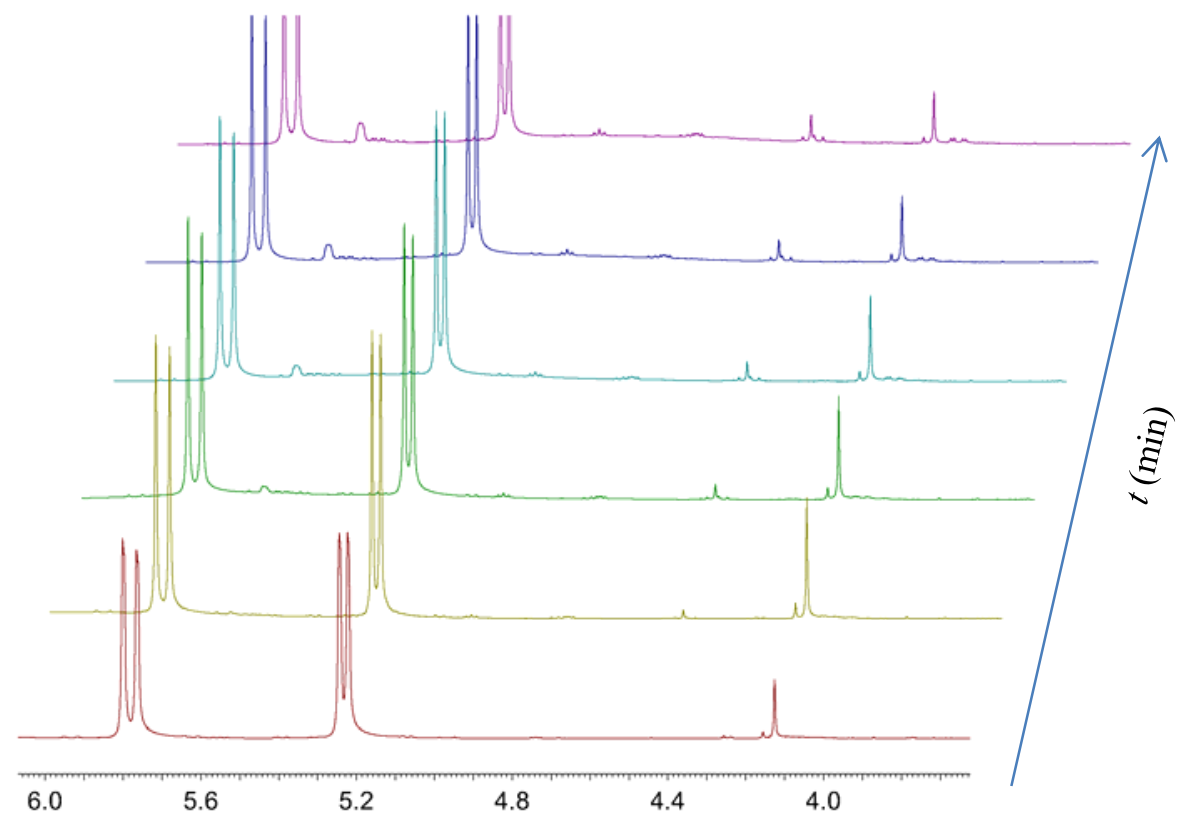

Figure S6. Selected ${ }^{1} \mathrm{H}$ NMR spectra monitored the oxidative [4+2] cycloaddition 


\section{Radical trapping experiment}

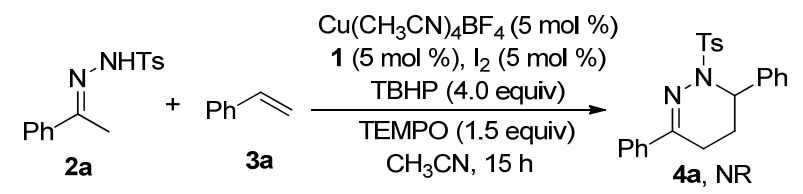

General procedure: To an oven-dried reaction tube was added $\mathrm{Cu}\left(\mathrm{CH}_{3} \mathrm{CN}\right)_{4} \mathrm{BF}_{4}$ (0.01 mmol, $5 \mathrm{~mol} \%)$ and diphenyl phosphate 1 (0.01 mmol, $5 \mathrm{~mol} \%)$. Distilled anhydrous $\mathrm{CH}_{3} \mathrm{CN}(0.5 \mathrm{~mL})$ was added. After stirring for $0.5 \mathrm{~h}$, sulfonyl hydrazine 2a (0.2 mmol, in $\left.0.4 \mathrm{~mL} \mathrm{CH}{ }_{3} \mathrm{CN}\right)$, styrene 3a (1.0 mmol), TEMPO (3.0 mmol), $\mathrm{I}_{2}(5$ $\mathrm{mol} \%$, in $\left.0.1 \mathrm{~mL} \mathrm{CH}_{3} \mathrm{CN}\right)$, and TBHP (0.8 mmol in decane) were added successively to the stirring reaction mixture. The mixture was stirred for 15 hours at room temperature, and no product 4a was obtained.

\section{Radical clock test}

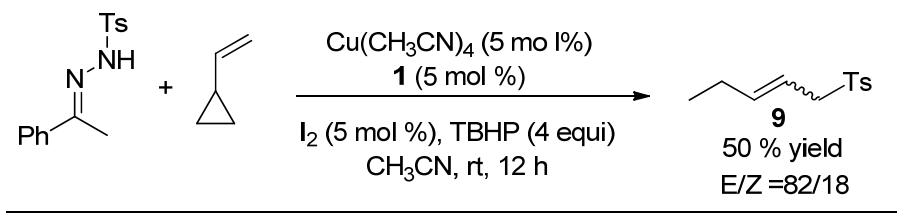

General procedure: To an oven-dried reaction tube was added $\mathrm{Cu}\left(\mathrm{CH}_{3} \mathrm{CN}\right)_{4} \mathrm{BF}_{4}$ (0.01 mmol, $5 \mathrm{~mol} \%)$ and diphenyl phosphate 1 (0.01 mmol, $5 \mathrm{~mol} \%)$. Distilled anhydrous $\mathrm{CH}_{3} \mathrm{CN}(0.5 \mathrm{~mL})$ was added. After stirring for $0.5 \mathrm{~h}$, sulfonyl hydrazine 2a (0.2 mmol, in $\left.0.4 \mathrm{~mL} \mathrm{CH}_{3} \mathrm{CN}\right)$, vinylcyclopropane (1.0 mmol), $\mathrm{I}_{2}(5 \mathrm{~mol} \%$, in $0.1 \mathrm{~mL}$ $\left.\mathrm{CH}_{3} \mathrm{CN}\right)$, and TBHP $(0.8 \mathrm{mmol}$ in decane) were added successively to the stirring reaction mixture. The mixture was stirred for 12 hours at room temperature, and product 9 was obtained, which was a known compound. ${ }^{4} 22.3 \mathrm{mg}$, yield: $50 \%, E / Z=$ 82/18. ${ }^{1} \mathrm{H}$ NMR (400 MHz, $\mathrm{CDCl}_{3}$, major- $\left.E\right): \delta 7.73(\mathrm{~d}, J=8.2 \mathrm{~Hz}, 2 \mathrm{H}), 7.33(\mathrm{~d}, J=$ 8.1, 2H), 5.59-5.52 (m, 1H), 5.43-5.36 (m, 1H), $3.72(\mathrm{~d}, J=7.3 \mathrm{~Hz}, 2 \mathrm{H}), 2.44(\mathrm{~s}, 3 \mathrm{H})$, 2.05-1.98 (m, $2 \mathrm{H}), 0.91(\mathrm{t}, J=7.5 \mathrm{~Hz}, 1 \mathrm{H}) \mathrm{ppm} ;{ }^{13} \mathrm{C} \mathrm{NMR}\left(100 \mathrm{MHz}, \mathrm{CDCl}_{3}\right): \delta$ $144.5,143.0,135.5,129.6,128.6,115.2,60.2,25.6,21.6,12.9$ ppm.

\section{Reference}

1. Hatcher, J. M.; Coltart, D. M. J. Am. Chem. Soc. 2010, 132, 4546.

2. Mori, H.; Takano, K.; Endo, T. Macromolecules 2009, 42, 7342. 
3. Zhao, X.; Zhang, L.; Li, T.; Liu, G.; Wang, H.; Lu, K. Chem. Commun. 2014, 50, 13121.

4. Inomata, K.; Sasaoka, S.-i.; Kobayashi, T.; Tanaka, Y.; Igarashi, S.; Ohtani, T.;

Kinoshita, H.; Kotake, H. Bull. Chem. Soc. Jap. 1997, 60, 1767-1780 


\section{NMR spectrum}
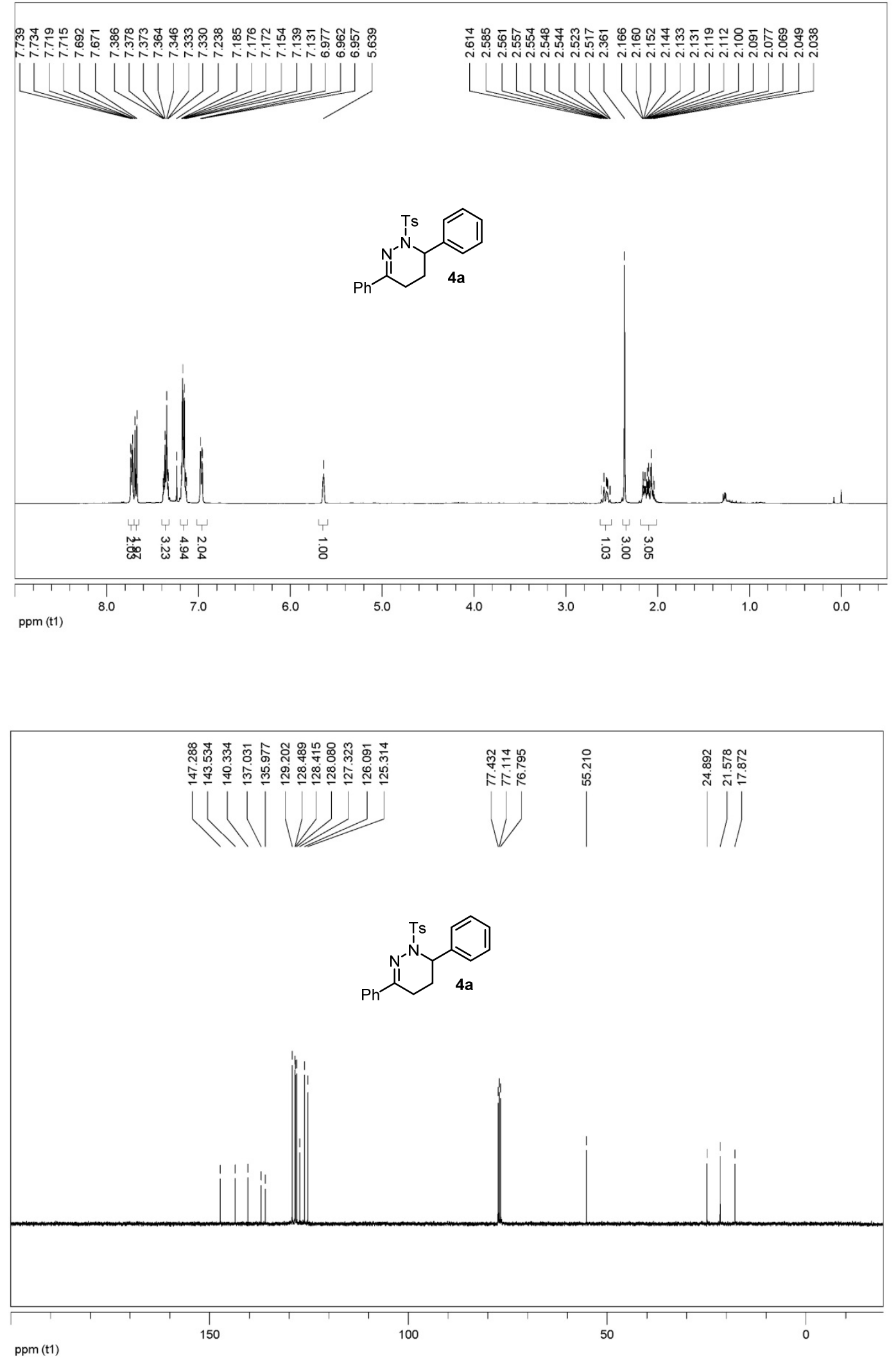

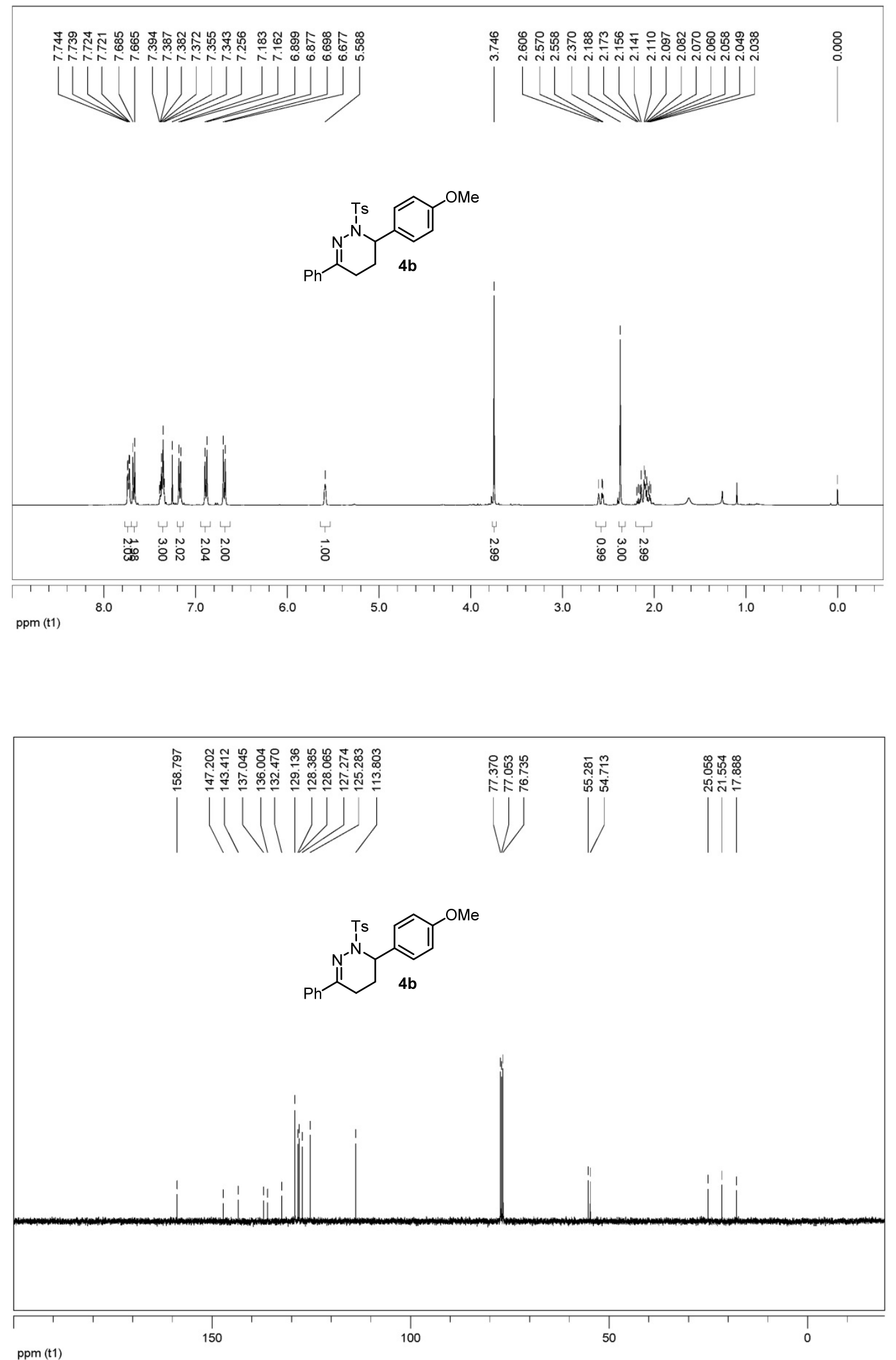

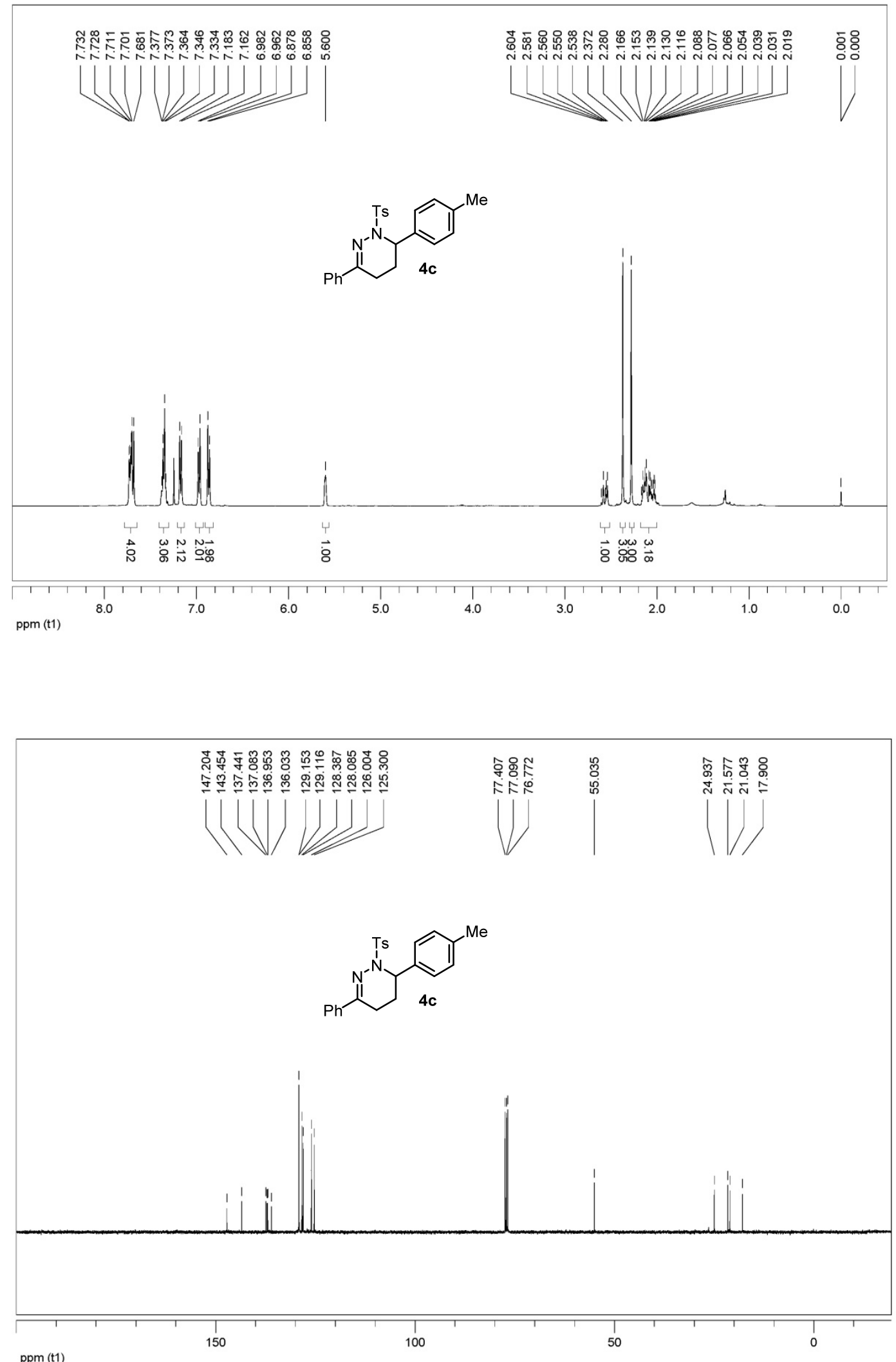

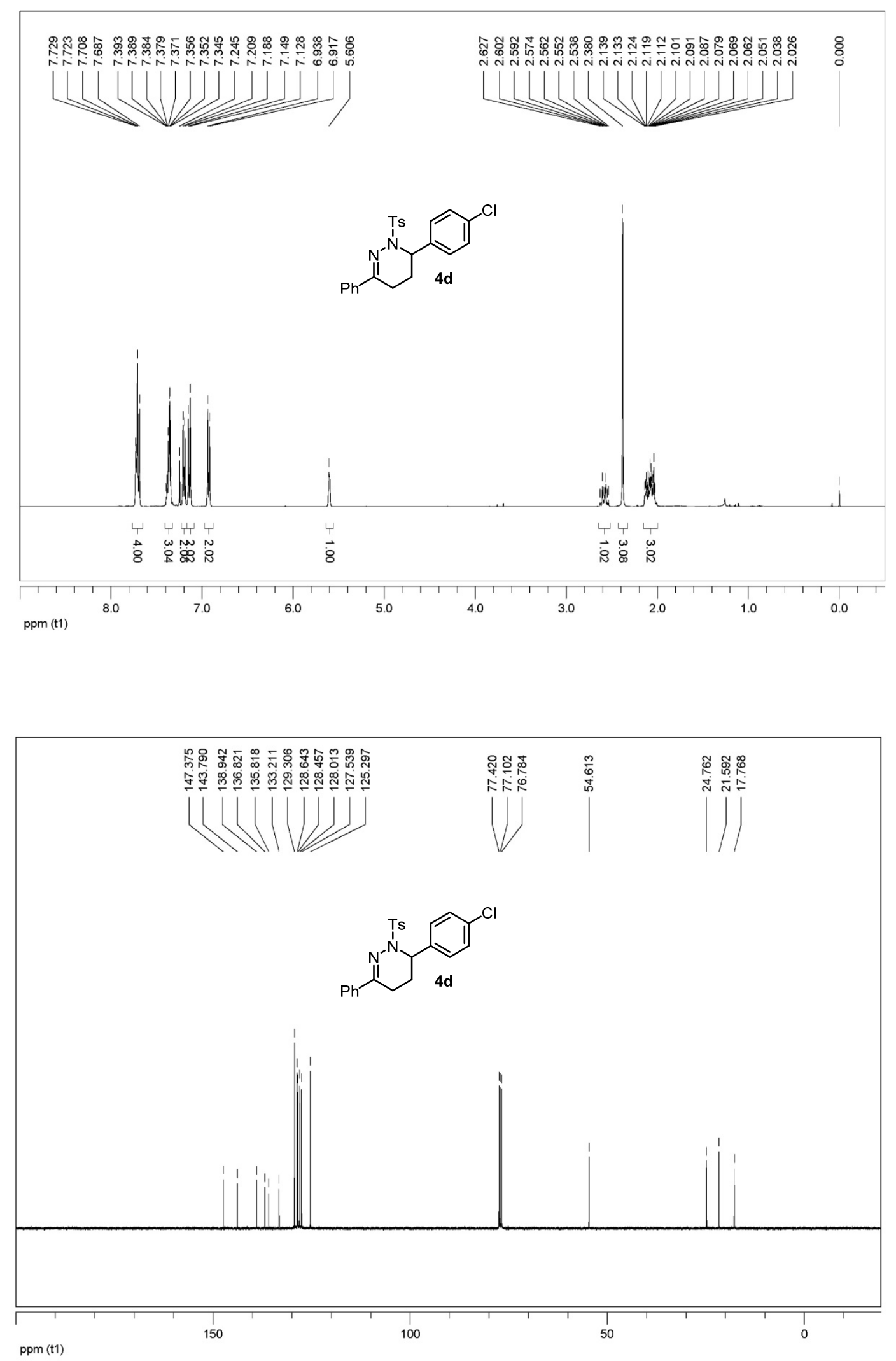

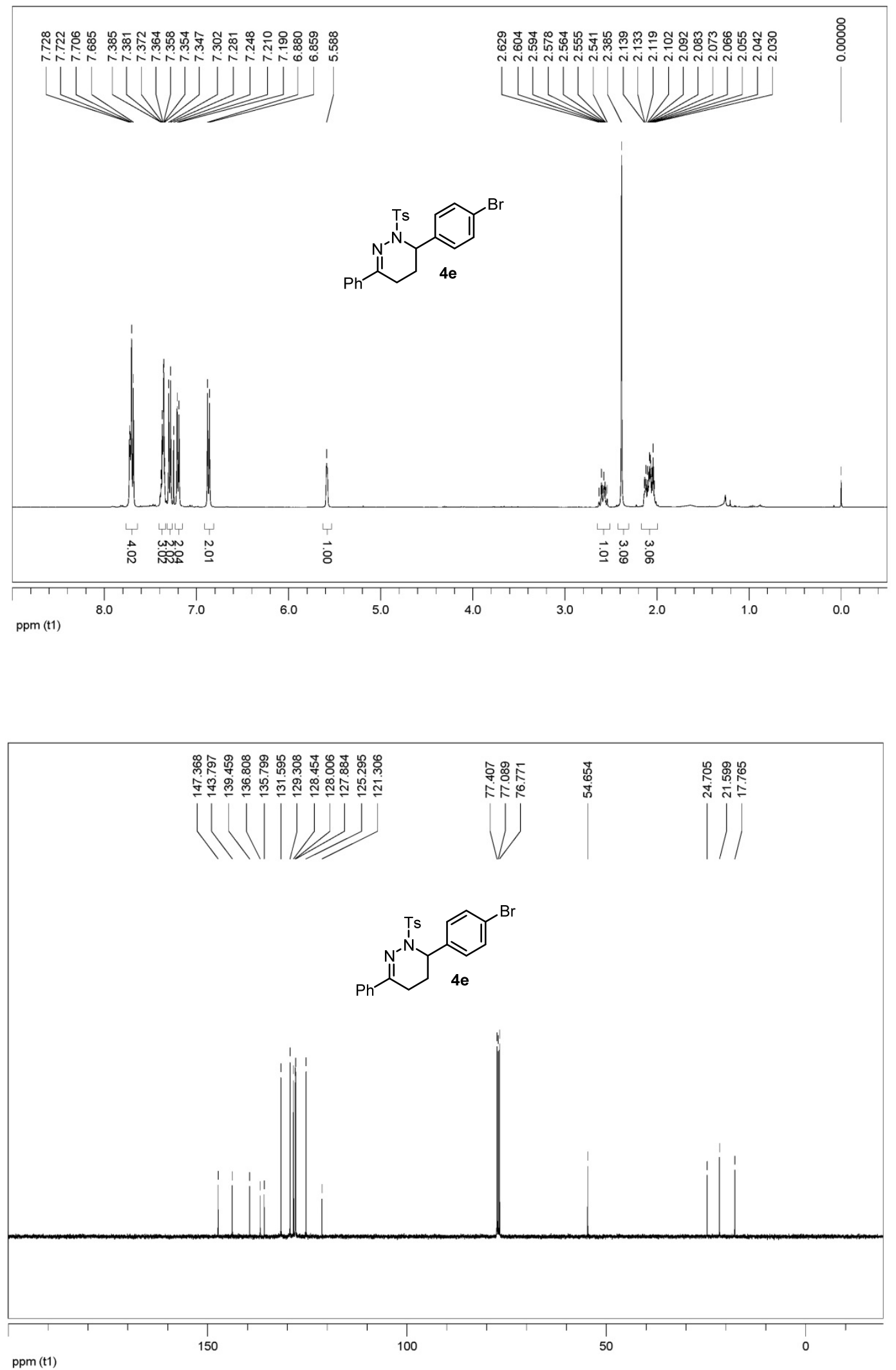

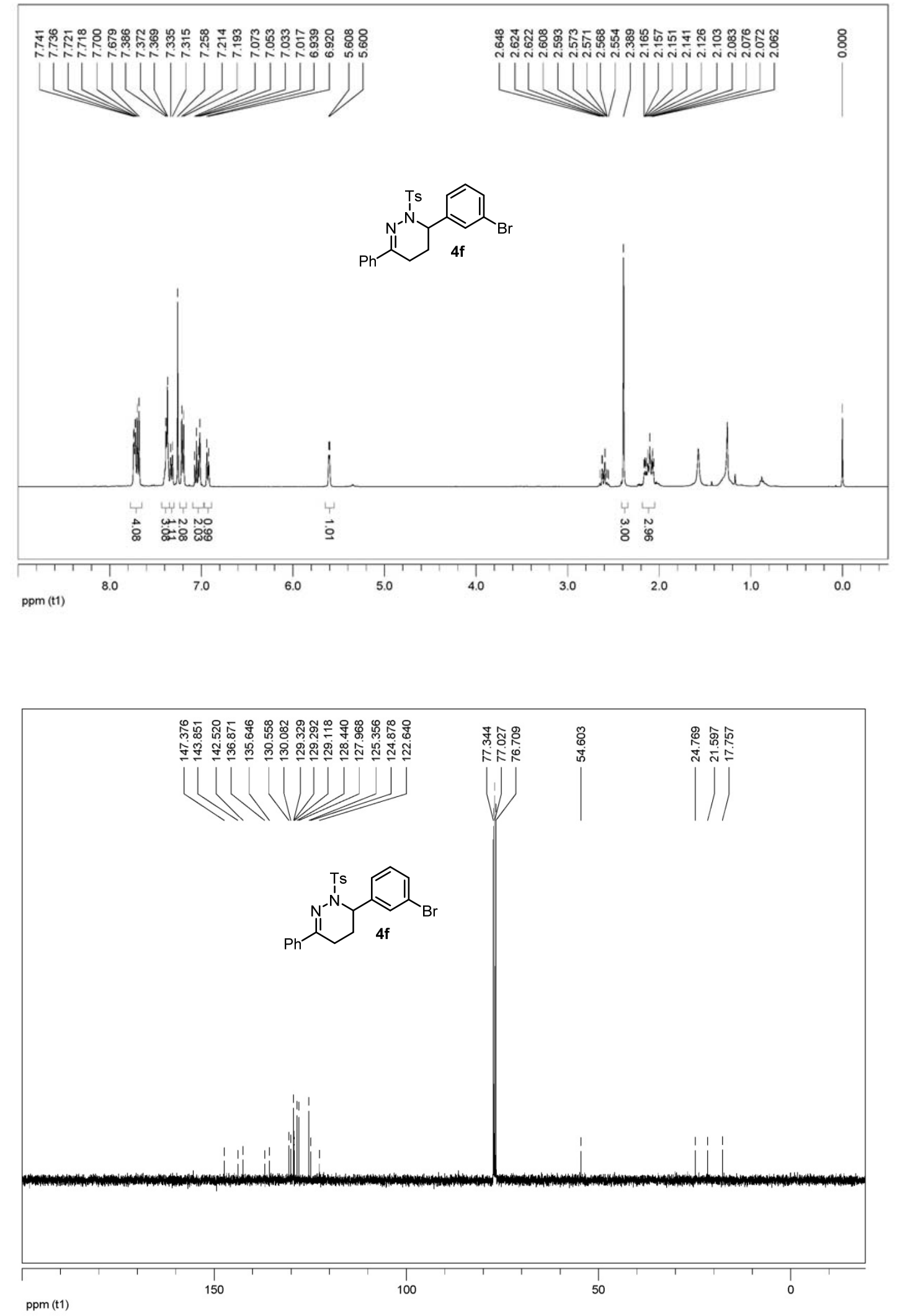


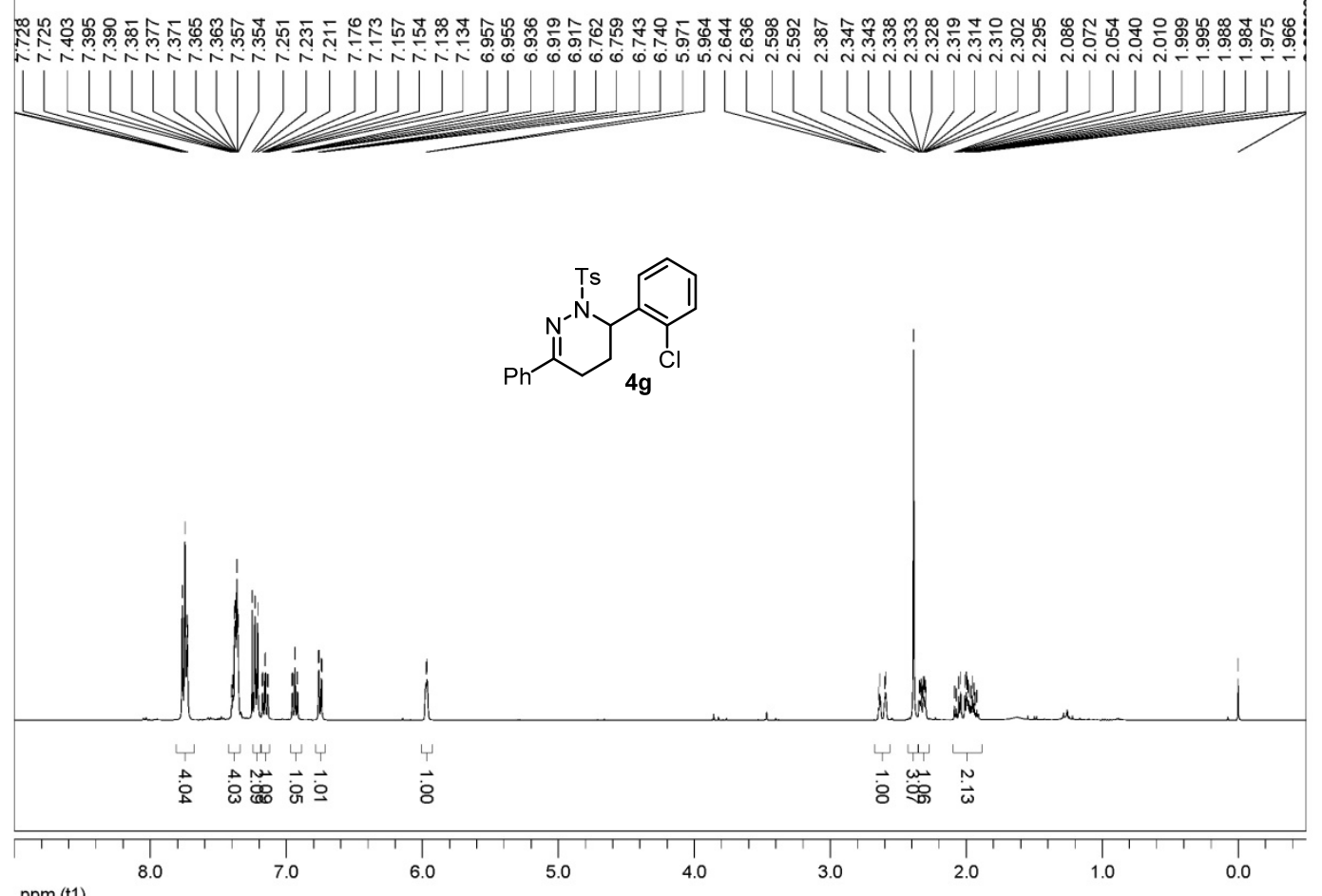

ppm (t1)

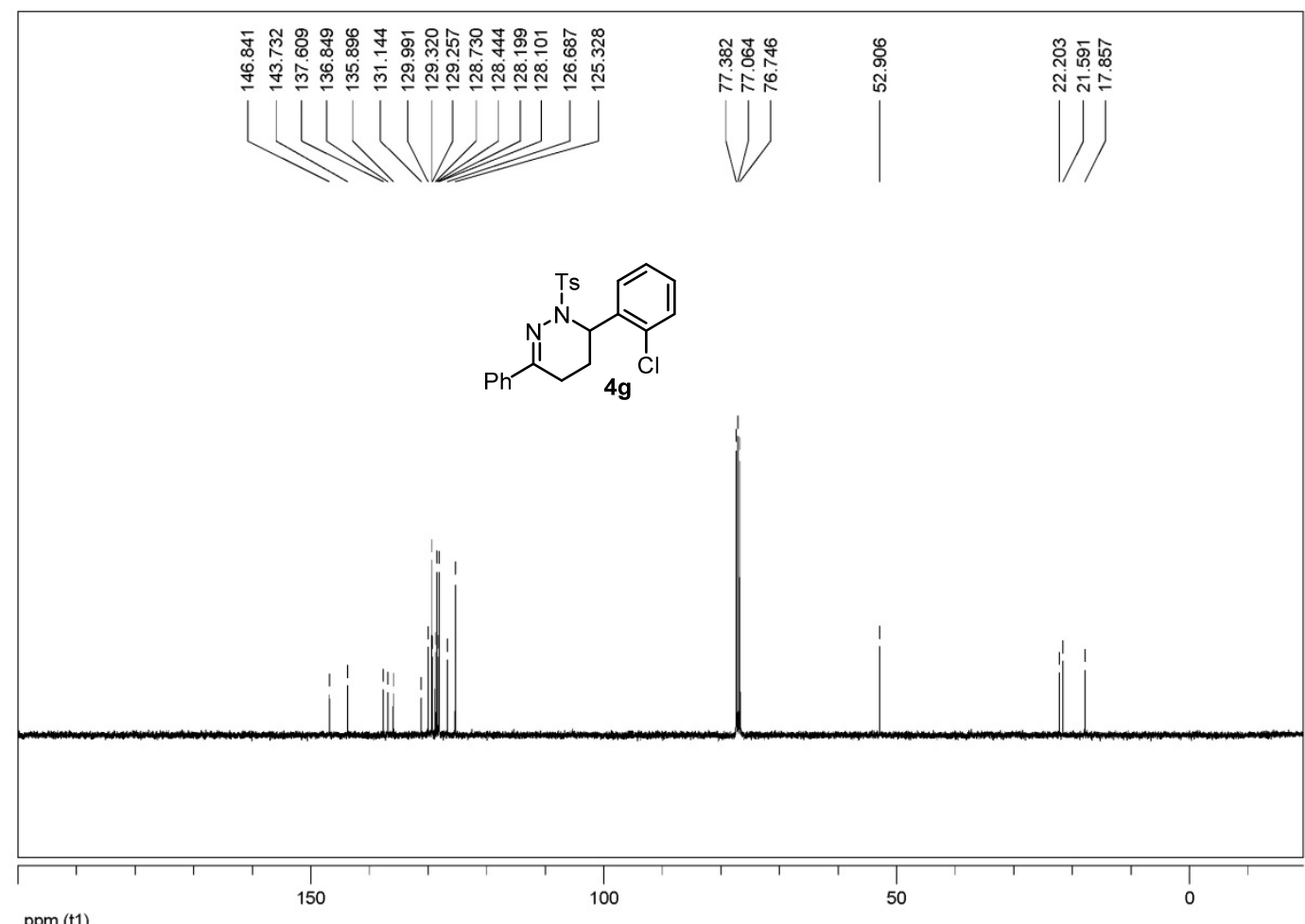



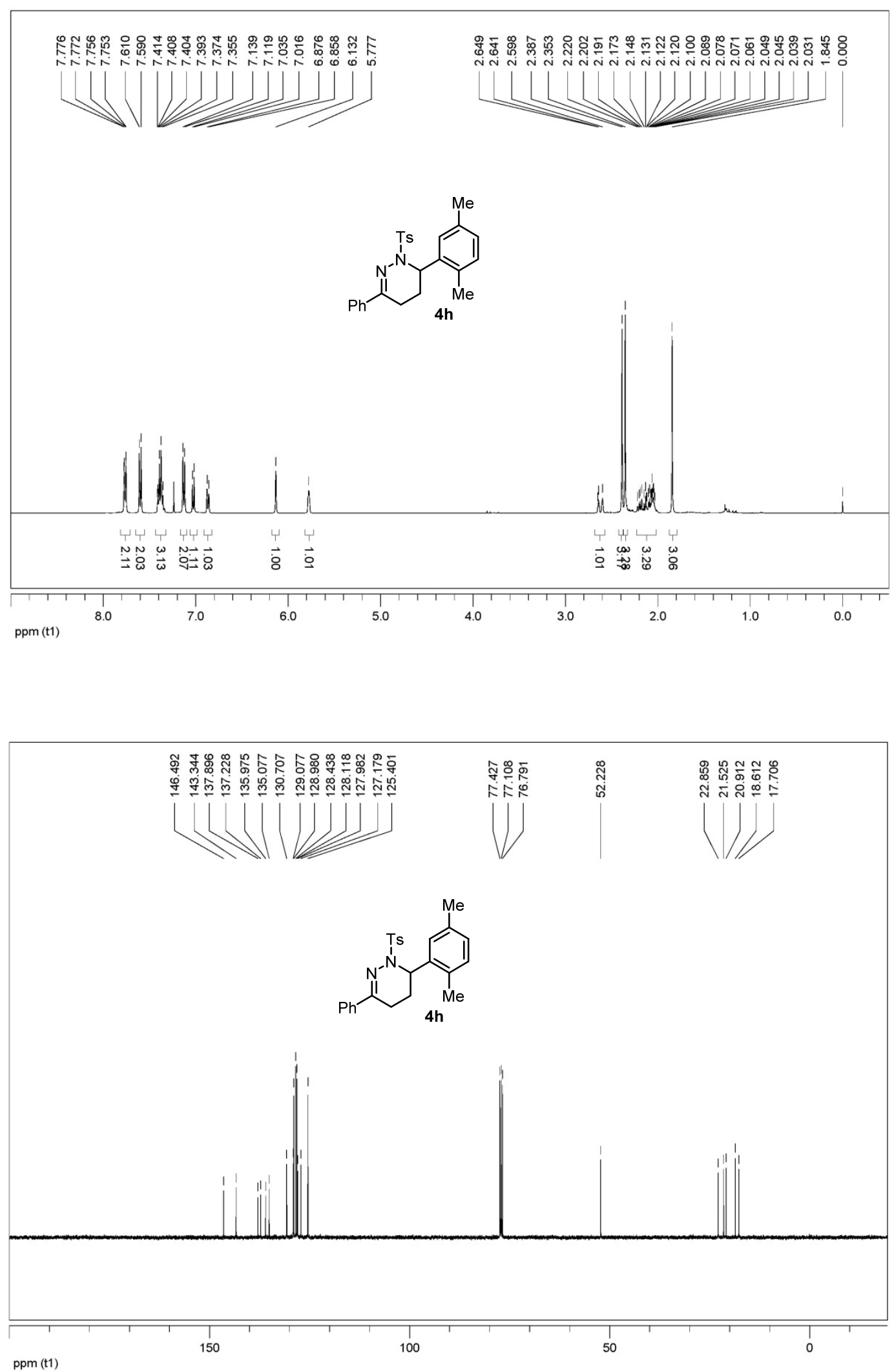

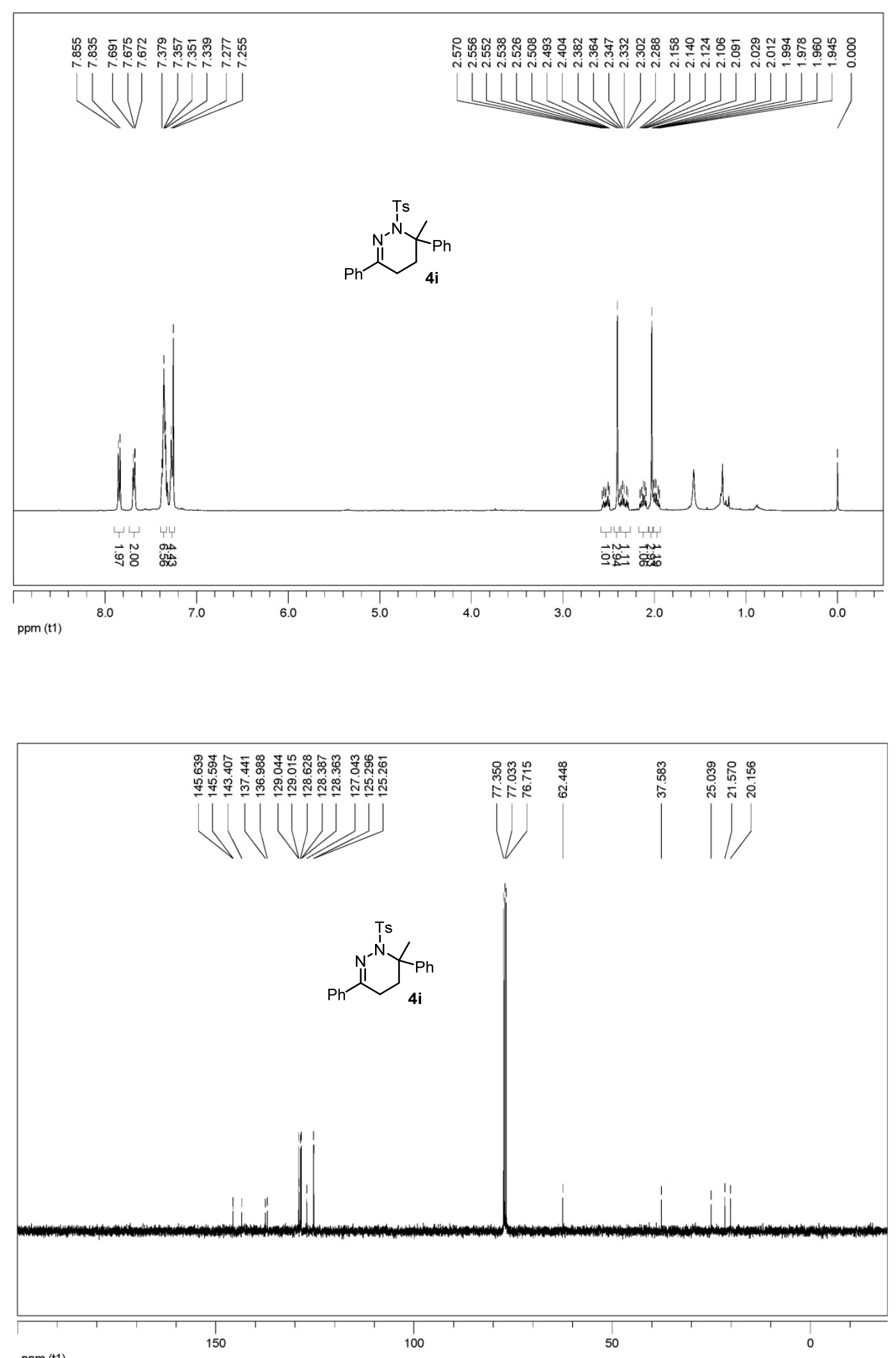

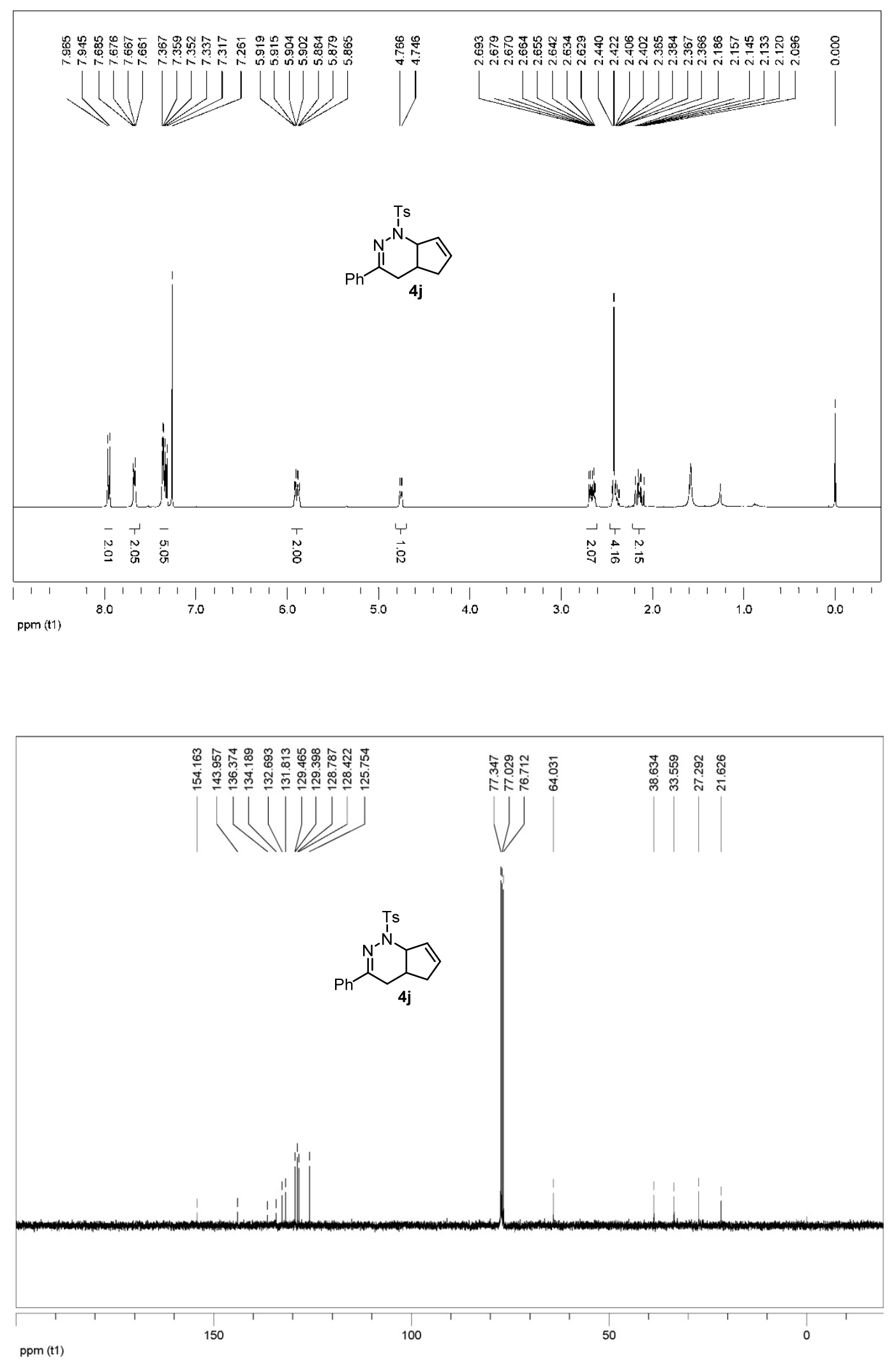

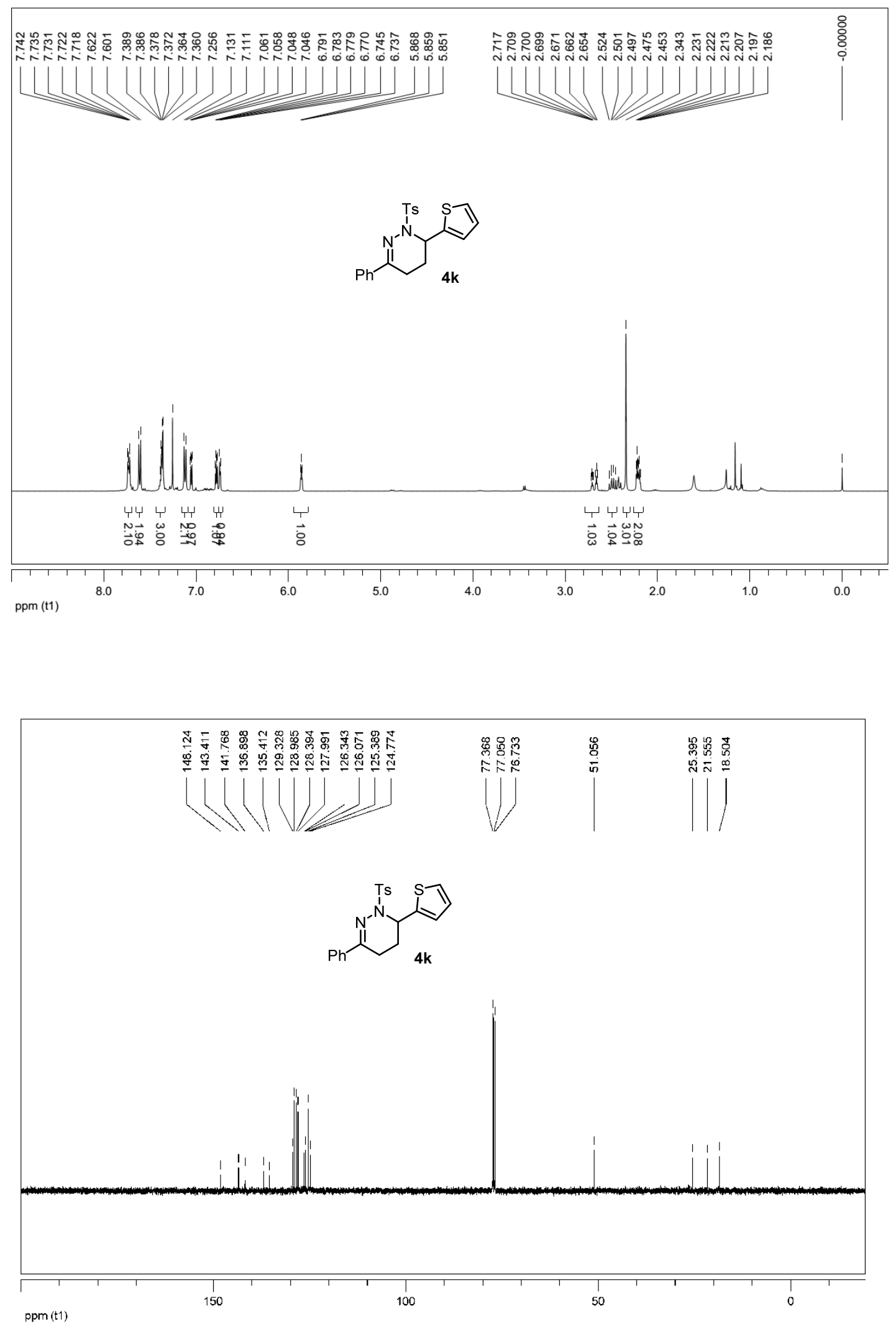


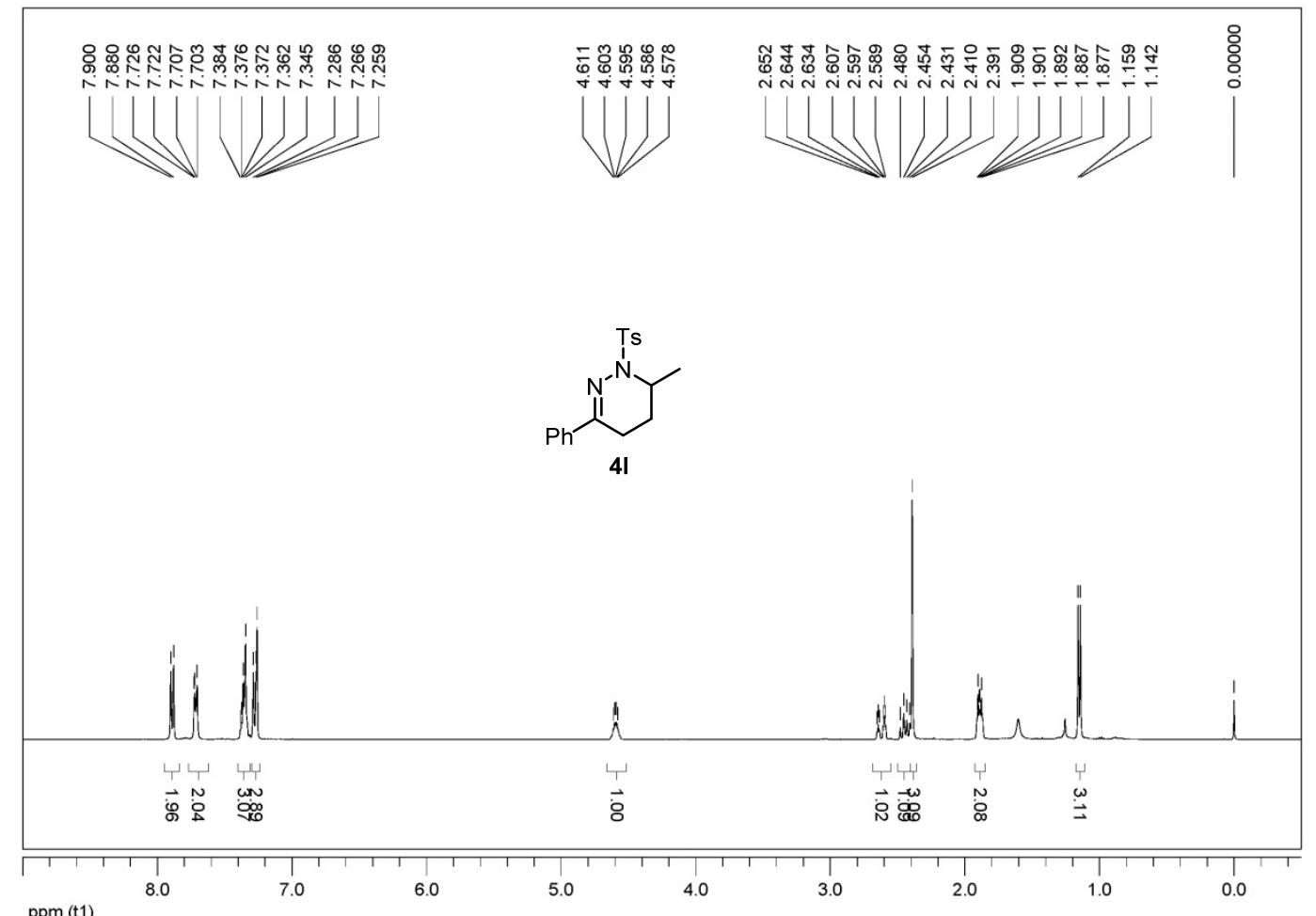

ppm (t

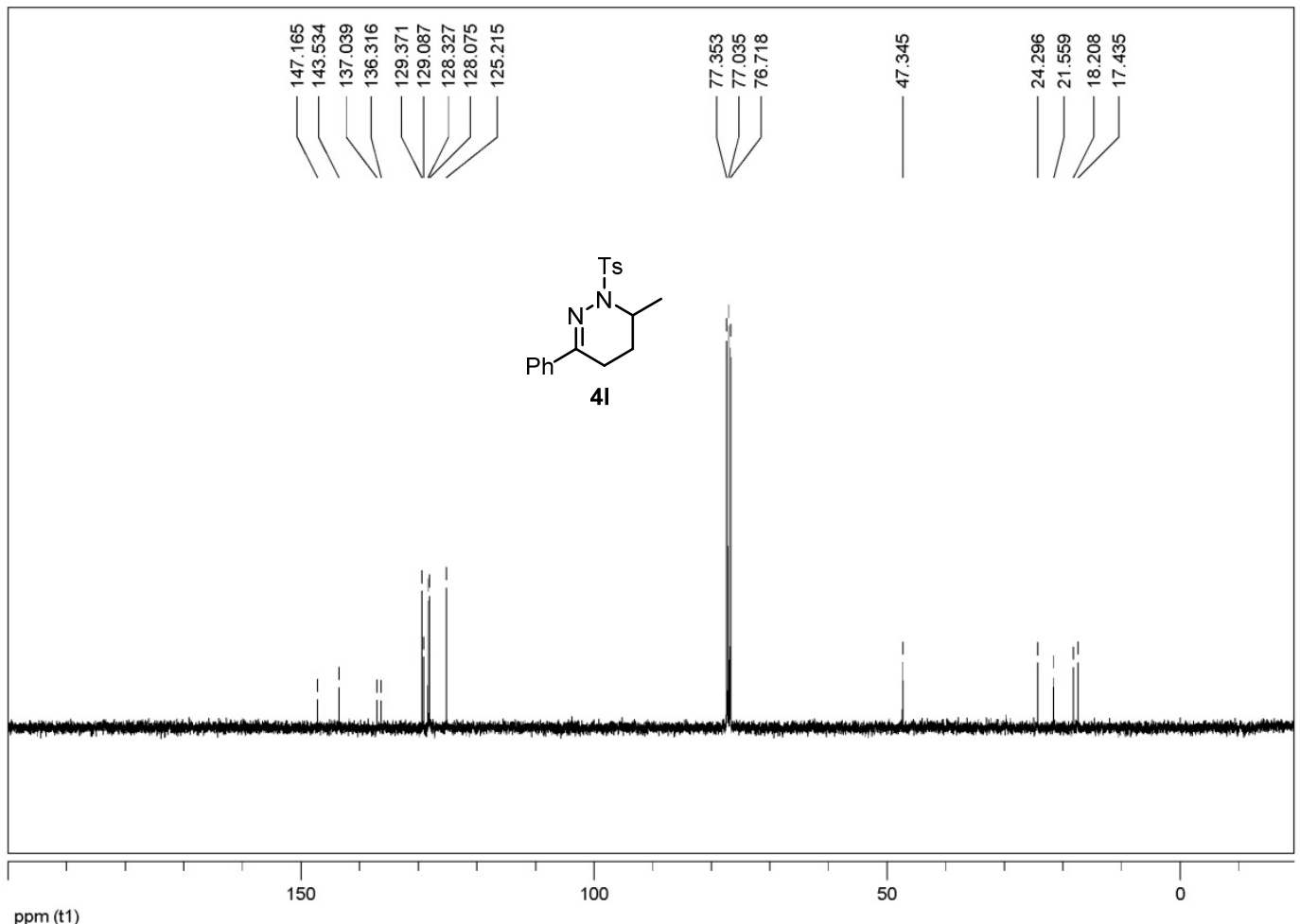



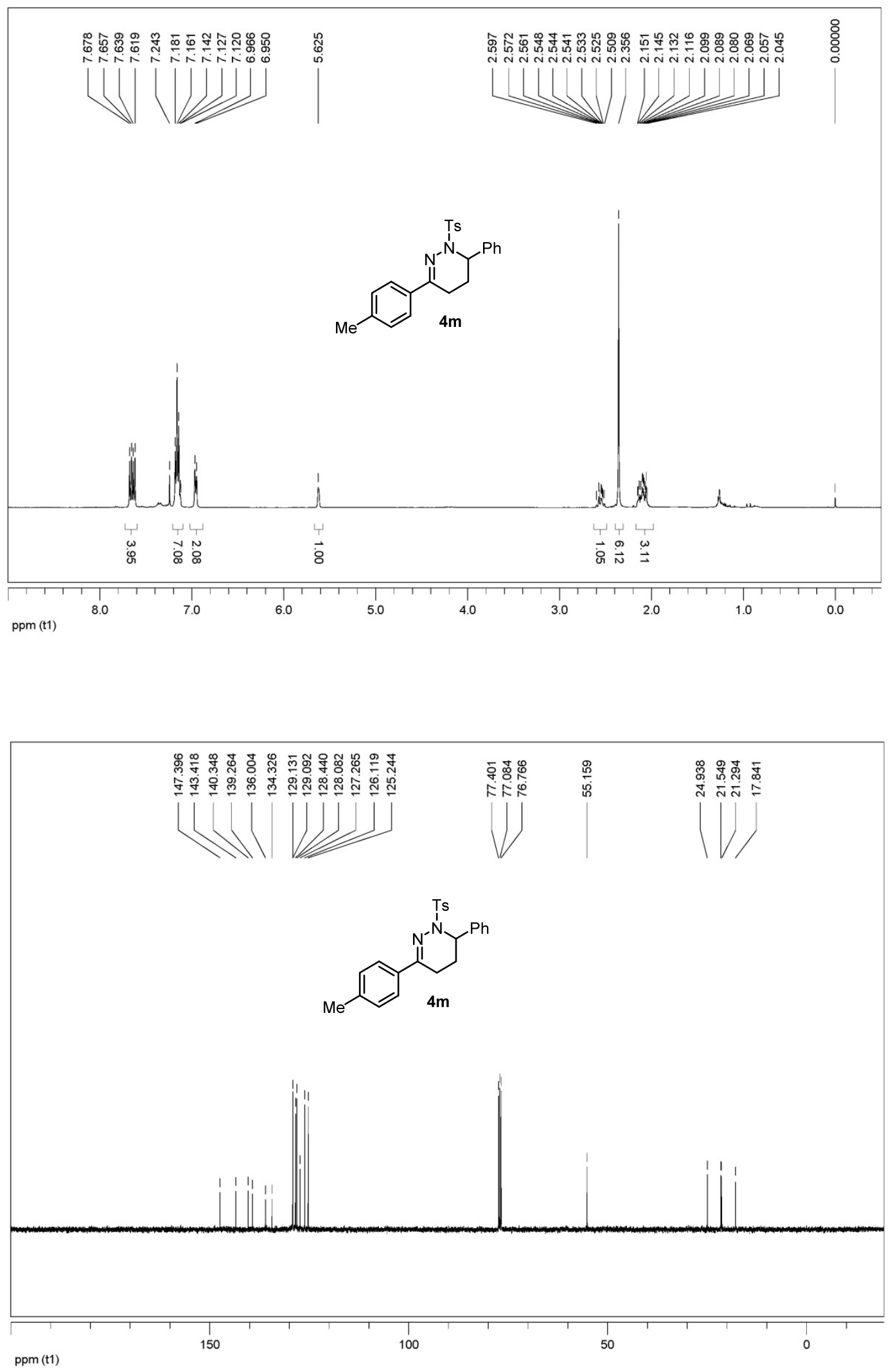

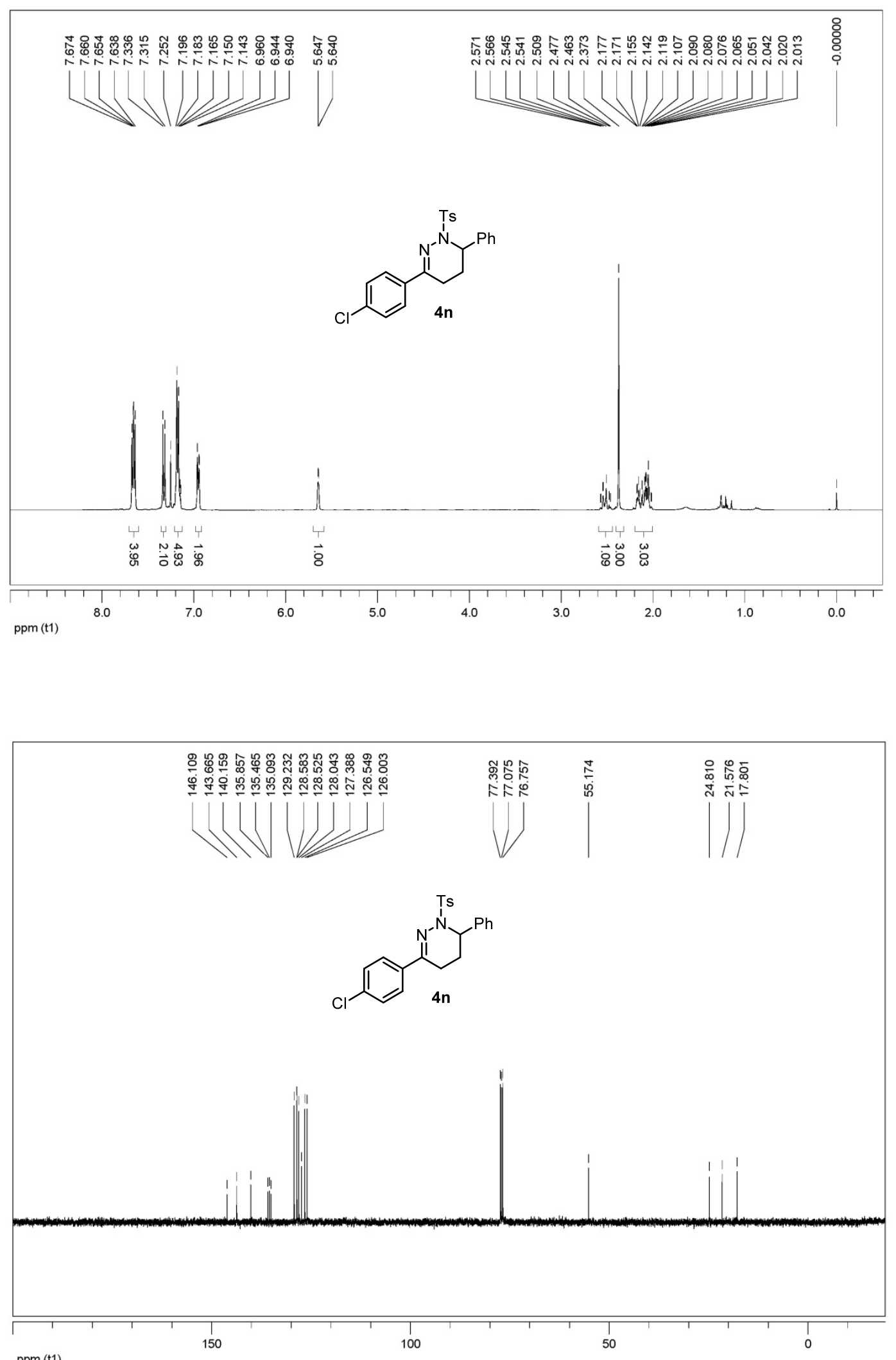

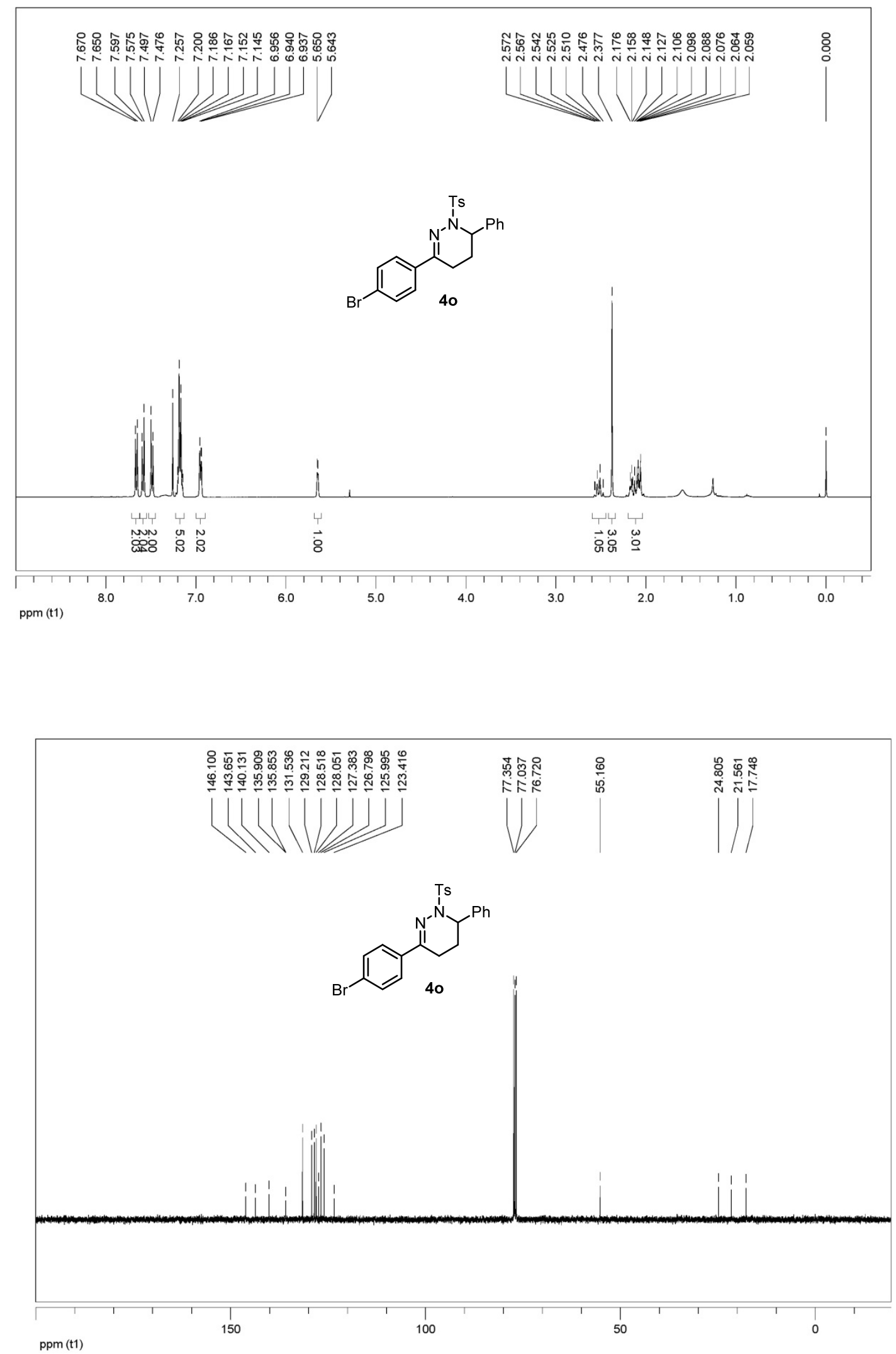

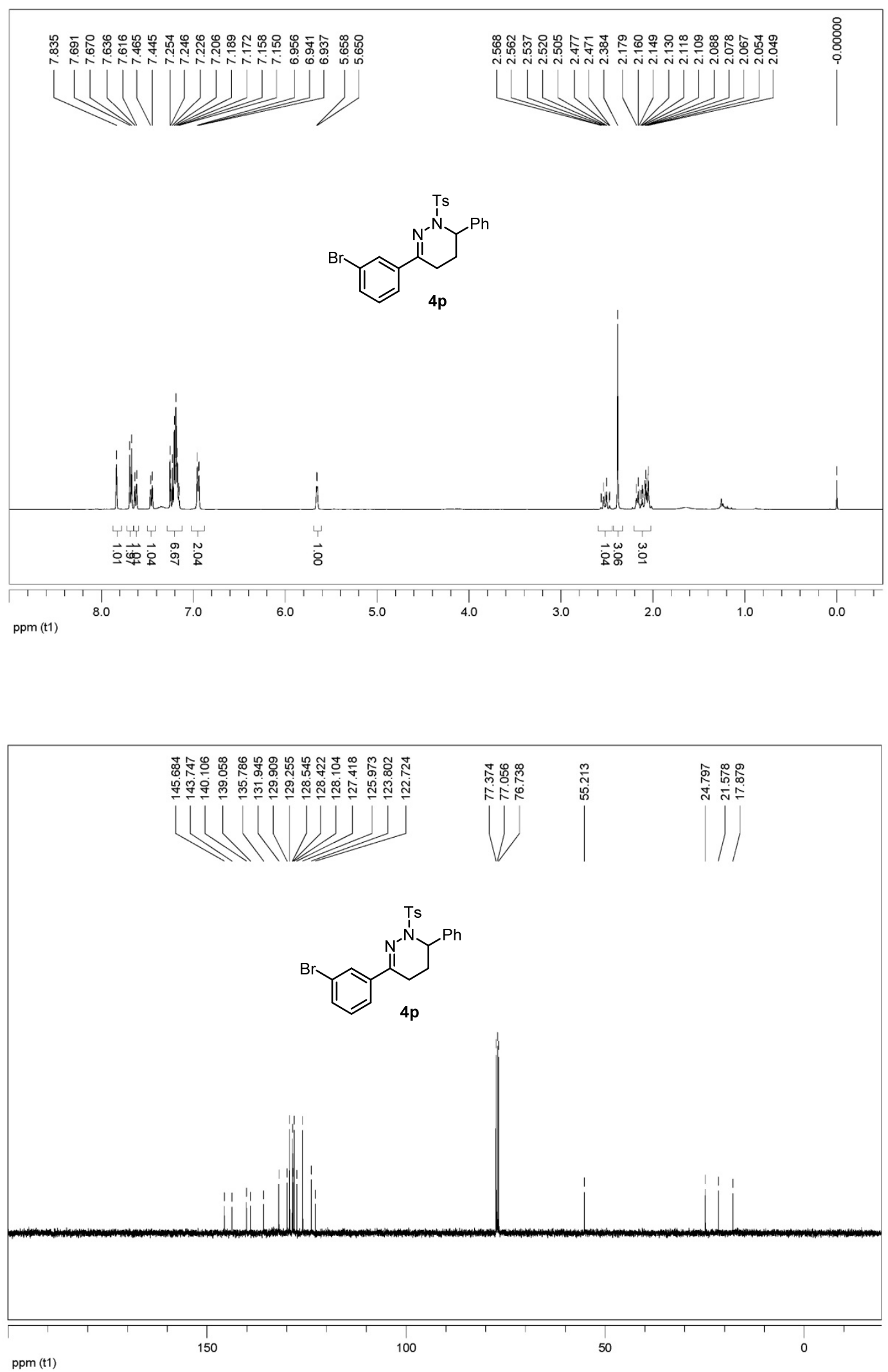

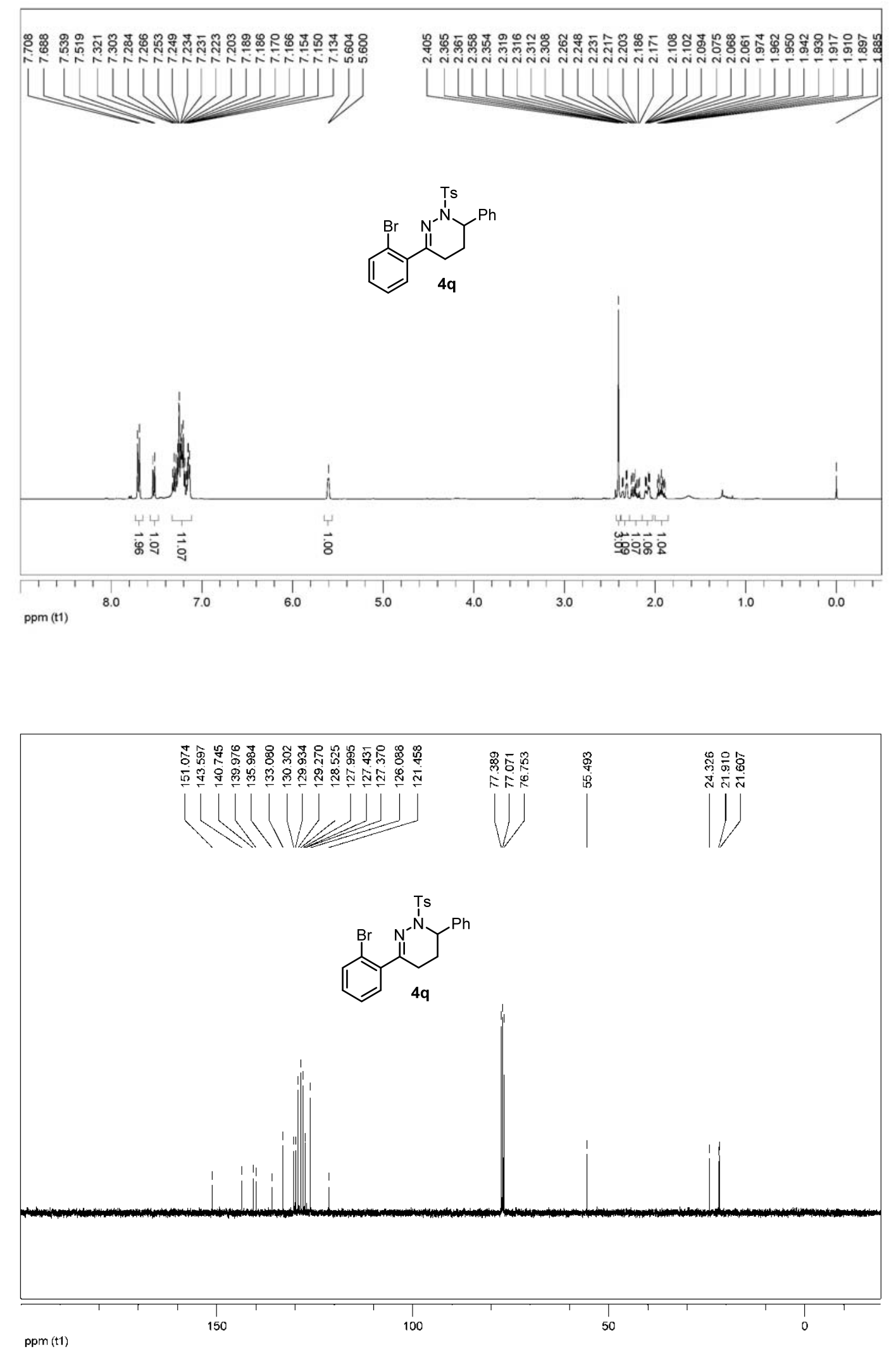


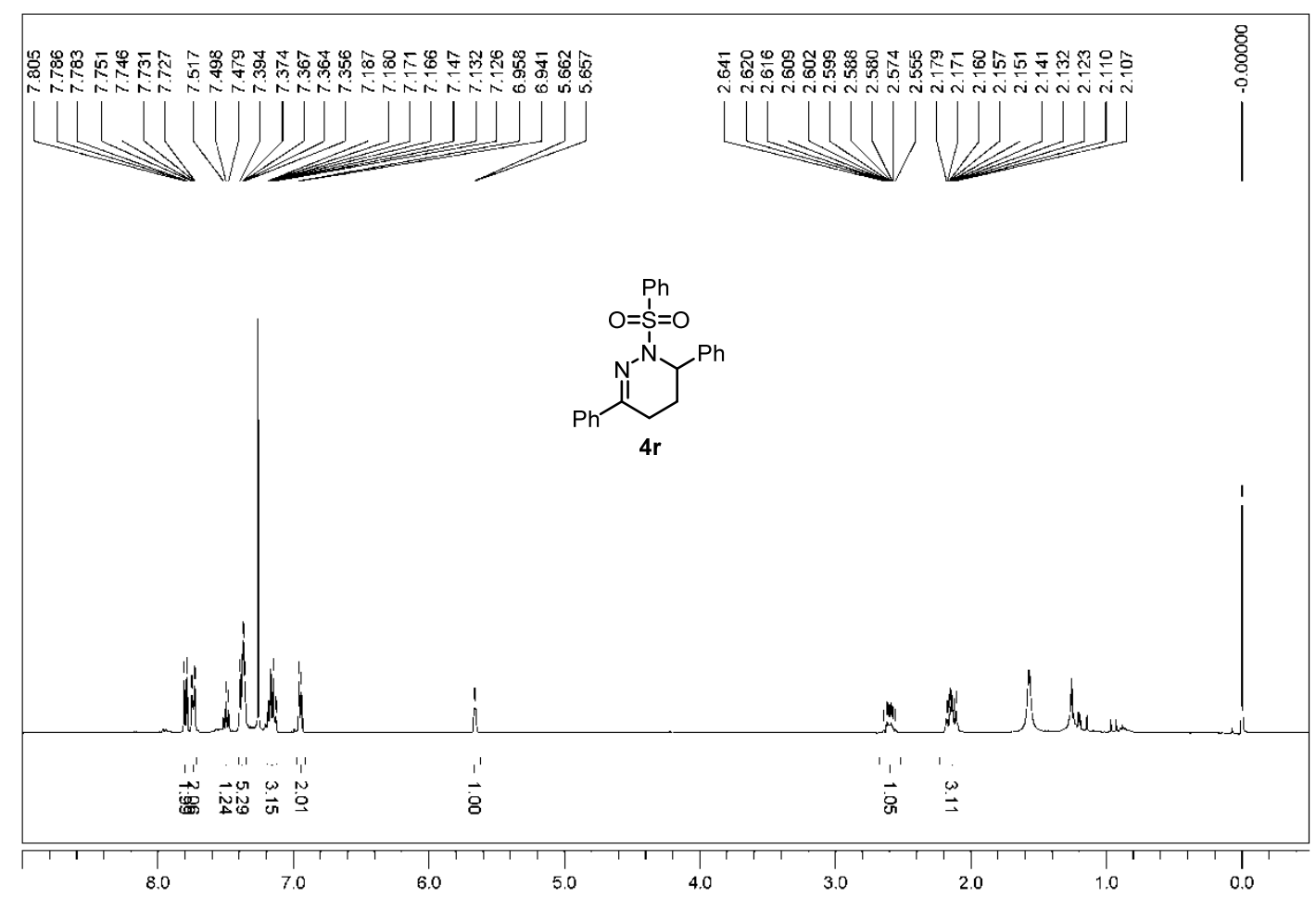

ppm (t1)

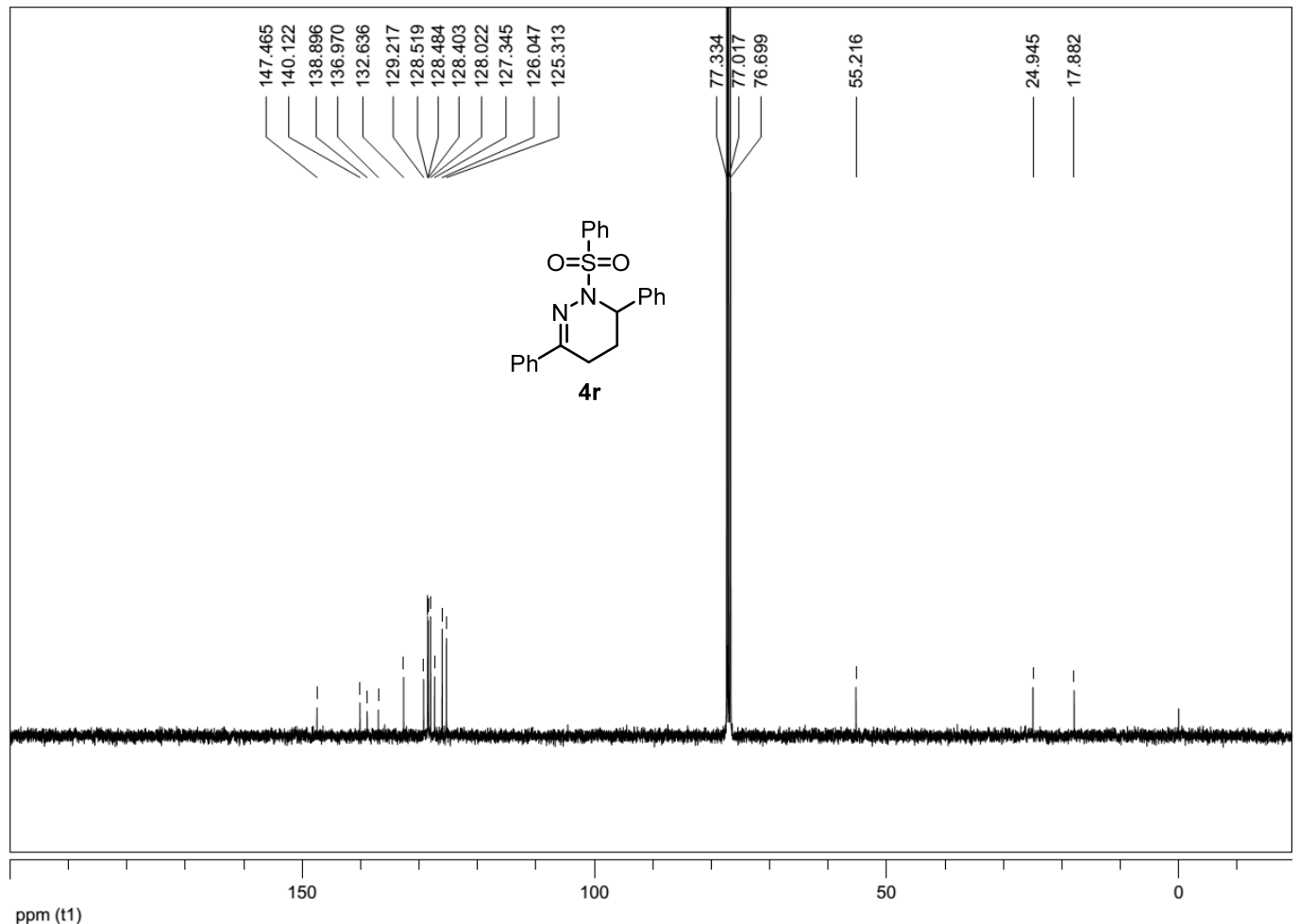



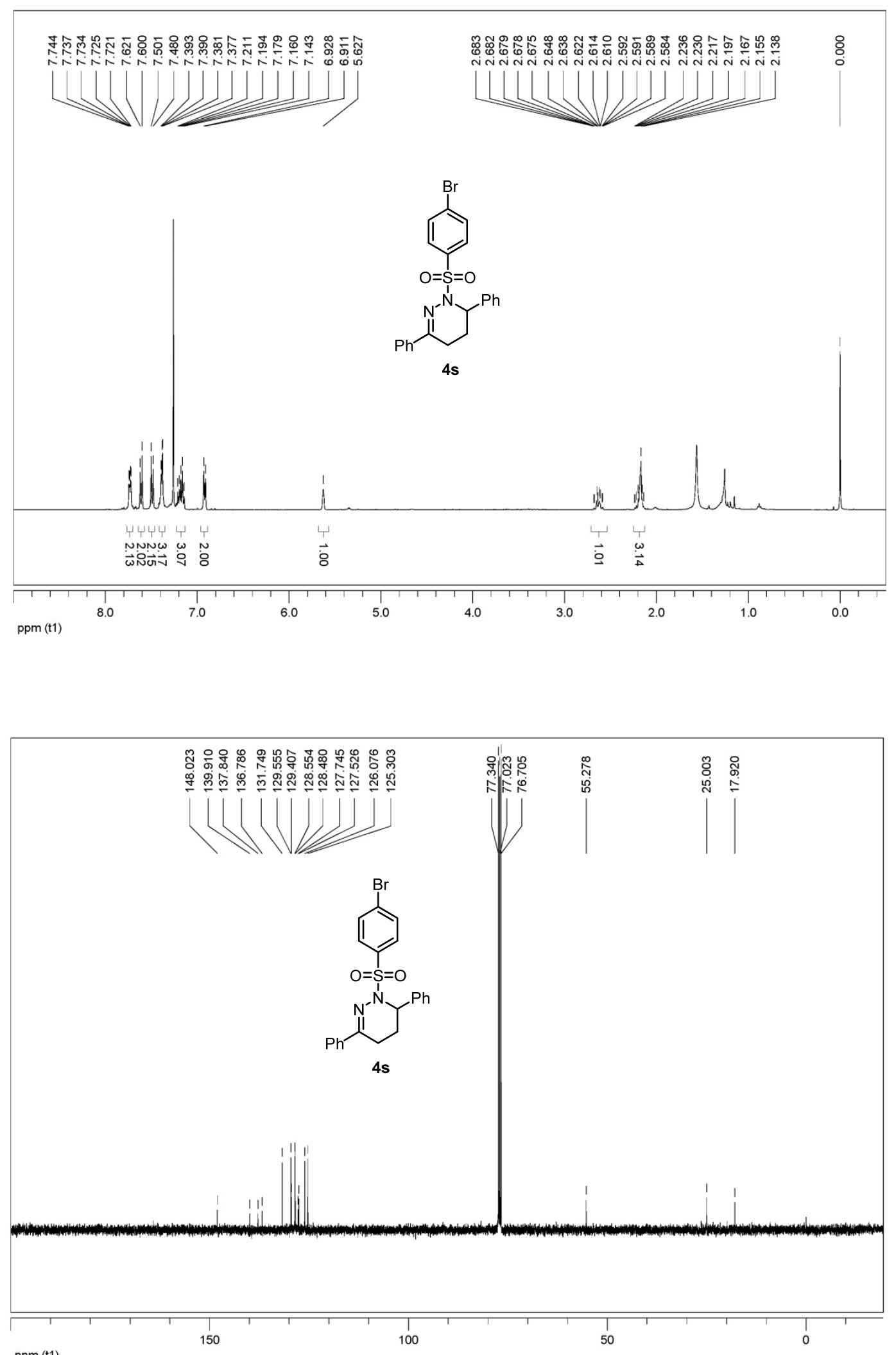

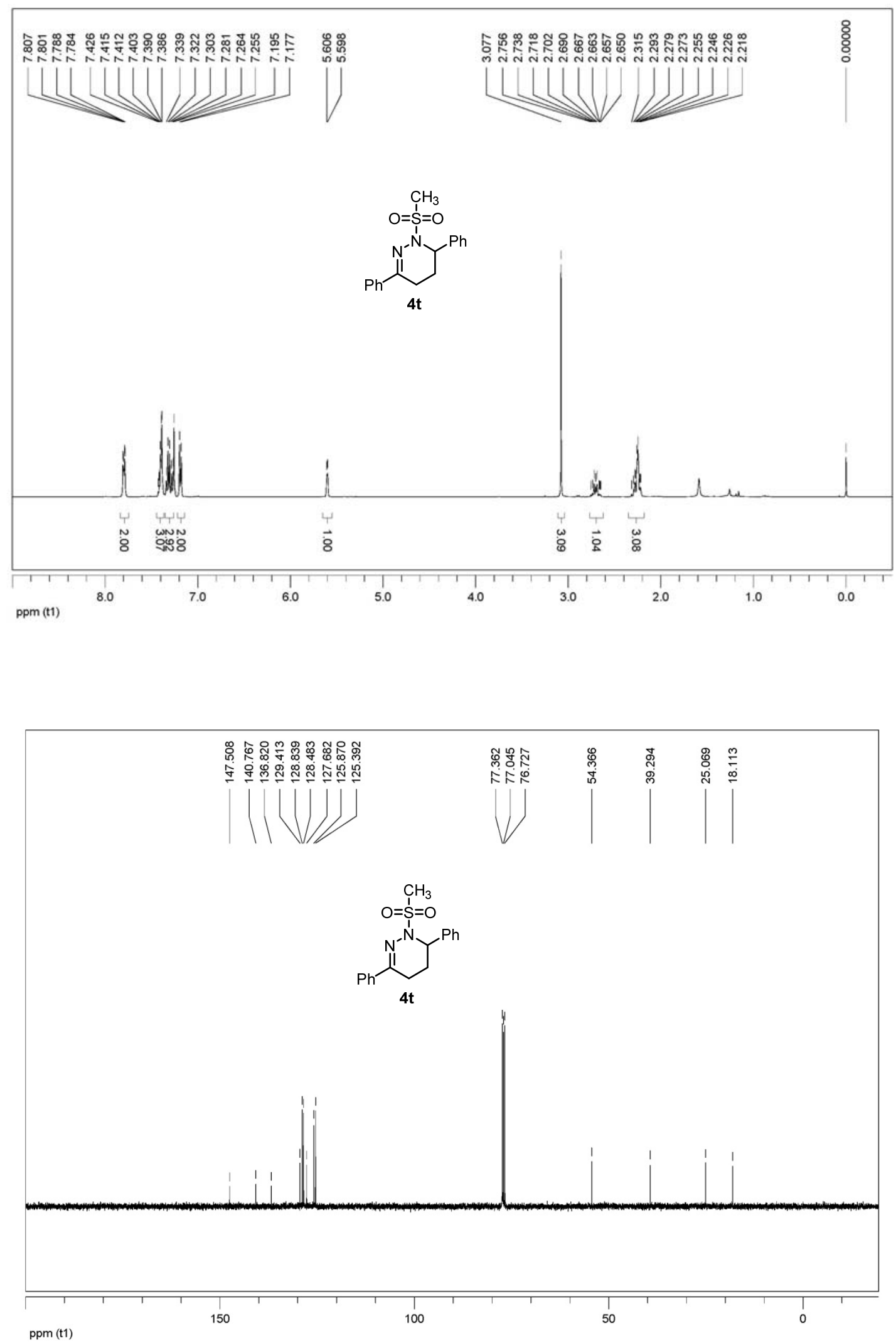

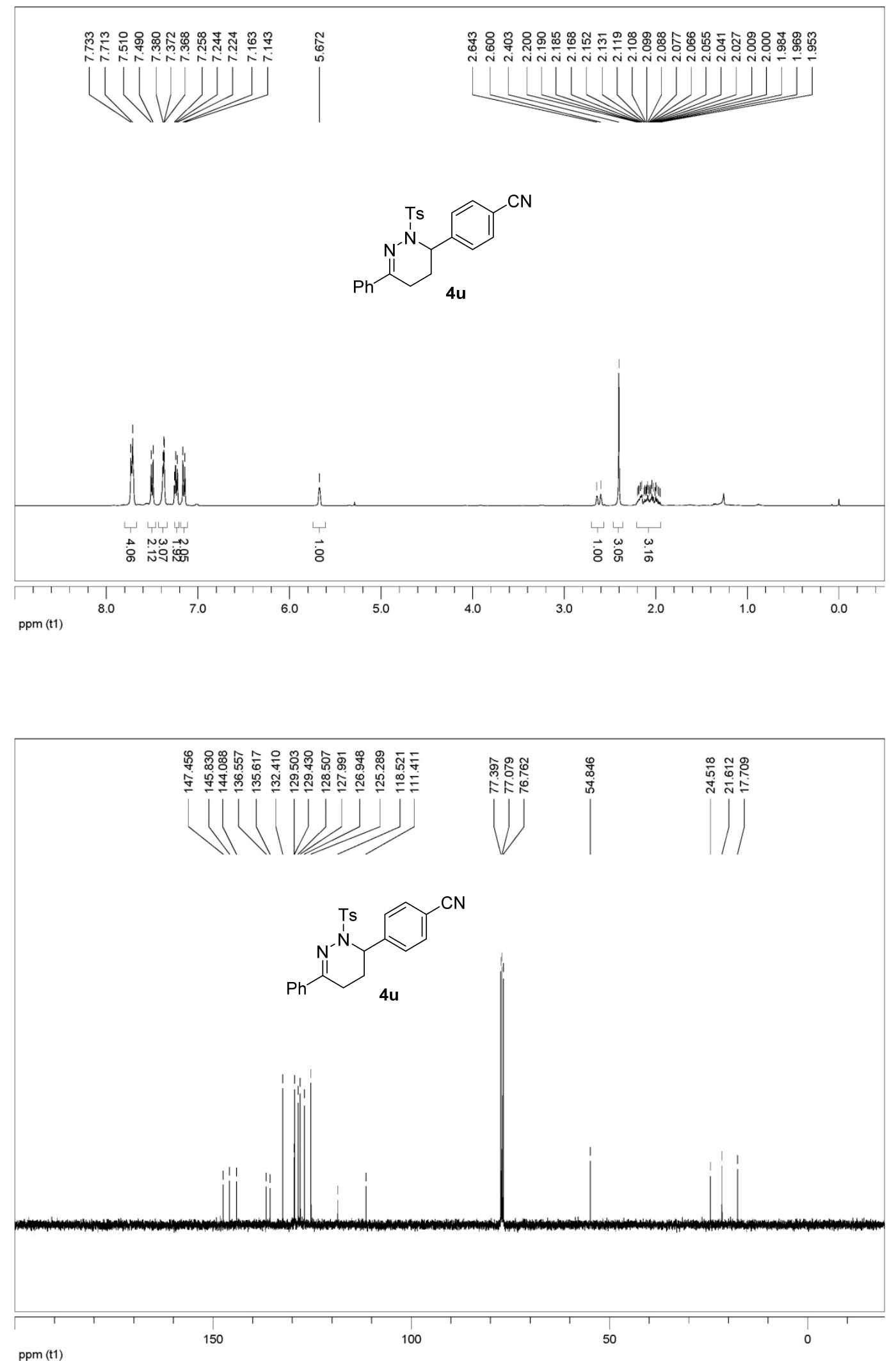

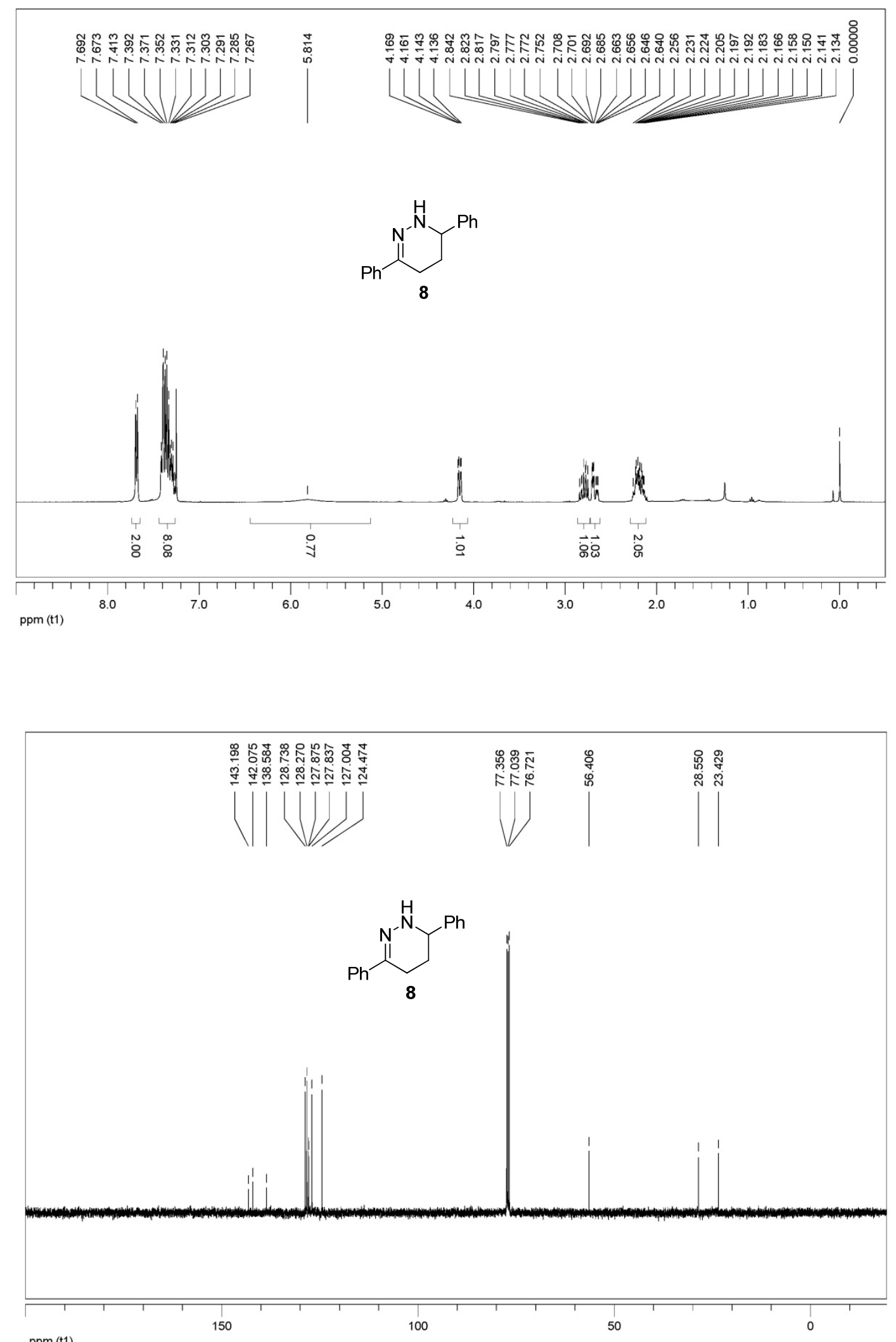